\title{
The fast Newtonian limit for
}

\section{perfect fluids}

\author{
Todd A. Oliynyk
}

School of Mathematical Sciences, Monash University, VIC 3800, Australia todd.oliynyk@monash.edu.au

\begin{abstract}
We prove the existence of a large class of dynamical solutions to the Einstein-Euler equations for which the fluid density and spatial threevelocity converge to a solution of the Poisson-Euler equations of Newtonian gravity. The results presented here generalize those of [10] to allow for a larger class of initial data. As in [10], the proof is based on a nonlocal symmetric hyperbolic formulation of the Einstein-Euler equations, which contain a singular parameter $\epsilon=v_{T} / c$ with $v_{T}$ a characteristic speed associated to the fluid and $c$ the speed of light. Energy and dispersive estimates on weighted Sobolev spaces are the main technical tools used to analyze the solutions in the singular limit $\epsilon \searrow 0$.
\end{abstract}

\section{Introduction}

The Einstein-Euler equations, which govern a gravitating perfect fluid, are given by

$$
G^{i j}=\frac{8 \pi G}{c^{4}} T^{i j} \quad \text { and } \quad \nabla_{i} T^{i j}=0
$$

e-print archive: http://lanl.arXiv.org/abs/0908.4455v2 
where

$$
T^{i j}=\left(\rho+c^{-2} p\right) v^{i} v^{j}+p g^{i j},
$$

with $\rho$ the fluid density, $p$ the fluid pressure, $v^{i}$ the fluid four-velocity normalized by $v^{i} v_{i}=-c^{2}, c$ the speed of light, and $G$ the Newtonian gravitational constant. Defining

$$
\epsilon=\frac{v_{T}}{c},
$$

where $v_{T}$ is a typical speed associated with the fluid, the Einstein-Euler equations, upon suitable rescaling [10], can be written in the form

$$
G^{i j}=2 \epsilon^{4} T^{i j} \quad \text { and } \quad \nabla_{i} T^{i j}=0,
$$

where

$$
T^{i j}=\left(\rho+\epsilon^{2} p\right) v^{i} v^{j}+p g^{i j} \quad \text { and } \quad v^{i} v_{i}=-\frac{1}{\epsilon^{2}} .
$$

In this formulation, the fluid four-velocity $v^{i}$, the fluid density $\rho$, the fluid pressure $p$, the metric $g_{i j}$, and the coordinates $\left(x^{i}\right) i=1, \ldots, 4$ are dimensionless. By assumption, the $\left(x^{i}\right)$ are global Cartesian coordinates on spacetime $M \cong \mathbb{R}^{3} \times[0, T)$, where the $\left(x^{I}\right)(I=1,2,3)$ are spatial coordinates that cover $\mathbb{R}^{3}$, and $t=x^{4} / v_{T}$ is a Newtonian time coordinate that covers the interval $[0, T)$. By a choice of units, we set $v_{T}=1$.

The Newtonian limit for the Einstein-Euler system refers to the limit of solutions of the Einstein-Euler systems in the limit $\epsilon \searrow 0$. In this limit, one expects that under reasonable assumptions solutions of the EinsteinEuler system should converge to a solution of the Poisson-Euler equations of Newtonian gravity:

$$
\begin{aligned}
\partial_{t} \tilde{\rho}+\partial_{I}\left(\tilde{\rho} \tilde{w}^{I}\right) & =0 \quad\left(\partial_{I}:=\partial_{x^{I}}\right), \\
\tilde{\rho}\left(\partial_{t} \tilde{w}^{J}+\tilde{w}^{I} \partial_{I} \tilde{w}^{J}\right) & =-\left(\tilde{\rho} \partial^{J} \tilde{\Phi}+\partial^{J} \tilde{p}\right) \quad\left(\partial^{J}:=\delta^{J I} \partial_{I}\right), \\
\Delta \tilde{\Phi} & =\tilde{\rho} \quad\left(\Delta:=\delta^{I J} \partial_{I} \partial_{J}\right),
\end{aligned}
$$

where $\tilde{\rho}, \tilde{p}$, and $\tilde{w}^{J}$ are the fluid density, pressure, and three-velocity, respectively.

The difficulty of analyzing the Newtonian limit arises from the fact that the limit $\epsilon \searrow 0$ is singular. The first general rigorous result on the Newtonian limit without any symmetry assumptions is [13]. There, it is shown that 
there exists a wide class of solutions to the Einstein equations coupled to Vlasov matter that have a well defined Newtonian limit as $\epsilon \searrow 0$. This work is based on an elliptic-hyperbolic formulation of the Einstein-Vlasov equations in a maximal slicing gauge. In [10], we used a different approach to prove existence of a large class of non-stationary solutions to the EinsteinEuler equations which have a Newtonian limit.

The main aim of this article is to establish the existence of a Newtonian limit for solutions to the Einstein-Euler equations under weaker conditions on the initial data as compared to either $[10,13]$. The purpose for this is twofold. First, it is of both theoretical and practical interest to understand the most general situations possible for which Newtonian gravity provides an acceptable approximation to full Einstein theory. Second, the techniques developed here can be used to improve the results of [11] on the existence of post-Newtonian expansions. In [11], it was shown that there exists a class of solutions to the Einstein-Euler equations that have a first post-Newtonian expansion. Using the methods here, this can be improved to the second post-Newtonian order. We will report on this in a separate article.

In this article, we follow the approach of [10] to analyze the limit $\epsilon \searrow 0$ of solutions to the Einstein-Euler equations. This requires that we replace the metric $g_{i j}$ and fluid velocity $v^{i}$ with new variables that are compatible with the limit $\epsilon \searrow 0$. The new gravitational variable is a density $\overline{\mathfrak{u}}^{i j}$ defined via the formula

$$
g^{i j}=\frac{\epsilon}{\sqrt{-\operatorname{det}(Q)}} Q^{i j}
$$

where

$$
Q^{i j}=\left(\begin{array}{cc}
\delta^{I J} & 0 \\
0 & 0
\end{array}\right)+\epsilon^{2}\left(\begin{array}{cc}
4 \overline{\mathfrak{u}}^{I J} & 0 \\
0 & -1
\end{array}\right)+4 \epsilon^{3}\left(\begin{array}{cc}
0 & \overline{\mathfrak{u}}^{I 4} \\
\overline{\mathfrak{u}}^{J 4} & 0
\end{array}\right)+4 \epsilon^{4}\left(\begin{array}{cc}
0 & 0 \\
0 & \overline{\mathfrak{u}}^{44}
\end{array}\right) .
$$

From these formulas, it not difficult to see that the density $\overline{\mathfrak{u}}^{i j}$ is equivalent to the metric $g_{i j}$ for $\epsilon>0$, and is well defined at $\epsilon=0$. For the fluid, a new velocity variable $w^{i}$ is defined by

$$
v^{I}=w^{I} \quad \text { and } \quad w^{4}=\frac{v^{4}-1}{\epsilon} .
$$

For technical reasons, we assume an isentropic equation of state

$$
p=K \rho^{(n+1) / n}
$$


for the fluid where $K \in \mathbb{R}_{>0}, n \in \mathbb{N}$. This allows us to use a technique of Makino [9] to regularize the fluid equations by the use of the fluid density variable $\alpha$ defined by

$$
\rho=\frac{1}{(4 K n(n+1))^{n}} \alpha^{2 n} .
$$

The resulting system can be put into a symmetric hyperbolic system that is regular across the fluid-vacuum interface. In this way, it is possible to construct solutions to the Einstein-Euler equations that represent compact gravitating fluid bodies (i.e., stars) both in the Newtonian and relativistic setting $[9,12]$.

The main point of introducing the gravitational-matter variables $\left\{\overline{\mathfrak{u}}^{i j}, w^{i}\right.$, $\alpha\}$ is that in a harmonic gauge the Einstein-Euler equations can be cast into a singular (non-local) symmetric hyperbolic system of the form

$$
b^{0}(\epsilon W) \partial_{t} W=\frac{1}{\epsilon} c^{I} \partial_{I} W+b^{I}(\epsilon, W) \partial_{I} W+F(\epsilon, W) .
$$

As shown in [10], for appropriately chosen initial data this form is suitable to derive $\epsilon$-independent energy estimates that can be use to analyze the behavior of the solutions as $\epsilon \searrow 0$, and extract a Newtonian limit. We note that singular hyperbolic systems of the form (1.10) have been extensively studied $[3,6,7,14,15]$, but, as discussed in [10], these results are not directly applicable to the Einstein-Euler equations due to initial data that does not lie in the standard Sobolev space $H^{k}\left(\mathbb{R}^{3}\right)$.

For general initial data, the $\epsilon$-independent energy estimates from [10] are not enough to control the solution in the limit $\epsilon \searrow 0$. In this paper, we show that when the energy estimates are used in conjunction with dispersive estimates for the wave equation a larger class of initial data can be chosen so that the resulting solutions still have a Newtonian limit. However, unlike the situation in [10], the gravitational variables do not converge to an $\epsilon$-independent limit. Instead, they converge to a solution of a singular $\epsilon$-dependent wave equation. Following the terminology used in other singular hyperbolic problems [15], we refer to this type of limit as a fast limit. We note that dispersive wave estimates have been used previously in a similar fashion to analyze the (singular) incompressible limit for the Euler equations $[5,16]$.

The precise statement of the existence of a Newtonian limit is contained in the following Theorem, which is the main result of this article. A proof can be found in Section 6. A definition of the weighted spaces 
$H_{\delta, \epsilon}^{k}\left(H_{\delta}^{k}:=H_{\delta, 1}^{k}\right)$ can be found in Appendix A of [10]. We also define $X_{T, s, k, \delta}:=\cap_{\ell=0}^{s+1} C^{\ell}\left([0, T), H_{\delta}^{k-\ell}\right)$.

Theorem 1.1. Suppose $-1<\delta<-1 / 2, s \in \mathbb{Z}_{\geq 2}, R>0, k \in \mathbb{Z}_{\geq 3+s}$, $\underset{o}{\alpha}$, $w^{I} \in H_{\delta-1}^{k}$, supp $\alpha \subset B_{R}, \mathfrak{z}^{I J} \in H_{\delta}^{k+1}, \mathfrak{z}_{4}^{I J} \in H_{\delta-1}^{k}$. Then there exists a $\stackrel{o}{T}>0, \epsilon_{0}>0$, and $\stackrel{o}{d}$ maps

$$
\begin{aligned}
& \overline{\mathfrak{u}}_{\epsilon}^{i j}(t)-\overline{\mathfrak{u}}_{\epsilon}^{i j}(0), \partial_{I} \overline{\mathfrak{u}}_{\epsilon}^{i j}(t), \partial_{t} \overline{\mathfrak{u}}_{\epsilon}^{i j}(t) \in X_{T, s, k, \delta-1} \quad 0<\epsilon \leq \epsilon_{0}, \\
& \tilde{\tilde{\mathfrak{u}}}_{\epsilon}^{i j}(t)-\overline{\mathfrak{u}}_{\epsilon}^{i j}(0), \partial_{I} \tilde{\tilde{\mathfrak{u}}}_{\epsilon}^{i j}(t), \partial_{t} \tilde{\overline{\mathfrak{u}}}_{\epsilon}^{i j}(t) \in X_{T, s, k, \delta-1} \quad 0<\epsilon \leq \epsilon_{0}, \\
& \rho_{\epsilon}(t), w_{\epsilon}^{i}(t) \in X_{T, s, k, \delta-1} \quad 0<\epsilon \leq \epsilon_{0}, \\
& \tilde{\rho}(t), \tilde{w}^{I}(t) \in X_{T, s, k, \delta-1}, \quad \tilde{\Phi}(t) \in X_{T, s, k+2, \delta} \quad \text { with } \quad \partial_{t} \tilde{\Phi}(t) \in X_{T, s, k+1, \delta-1},
\end{aligned}
$$

such that

(i) the triple $\left\{\overline{\mathfrak{u}}_{\epsilon}^{i j}(x, t), \rho_{\epsilon}(x, t), w_{\epsilon}^{i}(x, t)\right\}$ determines a solution to the Einstein-Euler (1.1) in the harmonic gauge for $0<\epsilon \leq \epsilon_{0}$ on the spacetime region $\left(x^{I}, t=x^{4}\right) \in D=\mathbb{R}^{3} \times[0, T)$ with Arnowitt-DeserMisner (ADM) mass given by

$$
m_{\mathrm{ADM}}=\int_{\mathbb{R}^{3}} \tilde{\rho}(x, 0) d x^{3}+\mathrm{O}\left(\epsilon^{2}\right),
$$

(ii) $\left\{\tilde{\rho}(x, t), \tilde{w}^{I}(x, t), \tilde{\Phi}(x, t)\right\}$ is a solution to the Poisson-Euler (1.2)-(1.4) with initial data $\left.\tilde{\rho}\right|_{t=0}=(4 K n(n+1))^{-1} \alpha_{o}^{2 n},\left.\tilde{w}^{I}\right|_{t=0}=\underset{o}{w^{I}}$,

(iii) $\tilde{\overline{\mathfrak{u}}}_{\epsilon}(x, t)$ is a solution to the wave equation

$$
\epsilon^{2} \partial_{t}^{2} \tilde{\overline{\mathfrak{u}}}_{\epsilon}^{i j}-\Delta \tilde{\overline{\mathfrak{u}}}_{\epsilon}^{i j}=-\delta_{4}^{i} \delta_{4}^{j} \tilde{\rho}+\epsilon^{2} \delta_{4}^{i} \delta_{4}^{j} \partial_{t}^{2} \tilde{\Phi},
$$

with initial conditions

$$
\begin{aligned}
\left.\tilde{\overline{\mathfrak{u}}}_{\epsilon}^{i j}\right|_{t=0} & =\delta_{I}^{i} \delta_{J}^{j} \mathfrak{z}^{I J}-2 \Delta^{-1} \partial_{I} \mathfrak{z}_{4}^{I J} \delta_{4}^{(i} \delta_{J}^{j)}+\delta_{4}^{i} \delta_{4}^{j}\left(\left.\tilde{\Phi}\right|_{t=0}+\Delta^{-1} \partial_{I J}^{2} \mathfrak{z}^{I J}\right), \\
\left.\partial_{t} \tilde{\overline{\mathfrak{u}}}_{\epsilon}^{i j}\right|_{t=0} & =\frac{1}{\epsilon}\left(\delta_{I}^{i} \delta_{J}^{j} \mathfrak{z}_{4}^{I J}-2 \partial_{I} \mathfrak{z}^{I J} \delta_{4}^{(i} \delta_{J}^{j)}+\delta_{4}^{i} \delta_{4}^{j} \Delta^{-1} \partial_{I J}^{2} \mathfrak{z}_{4}^{I J}\right)+\left.\delta_{4}^{i} \delta_{4}^{j} \partial_{t} \tilde{\Phi}\right|_{t=0},
\end{aligned}
$$

(iv)

$$
\left\|\rho_{\epsilon}(t)-\tilde{\rho}(t)\right\|_{H^{k-2}}+\left\|w_{\epsilon}^{I}(t)-\tilde{w}^{I}(t)\right\|_{H^{k-2}}+\left\|w_{\epsilon}^{4}(t)\right\|_{H^{k-2}} \lesssim \epsilon,
$$

and

$$
\begin{aligned}
& \left\|\overline{\mathfrak{u}}_{\epsilon}^{i j}(t)-\tilde{\overline{\mathfrak{u}}}_{\epsilon}^{i j}(t)\right\|_{L^{6}}+\left\|\partial_{I} \overline{\bar{u}}_{\epsilon}^{i j}(t)-\partial_{I} \tilde{\overline{\mathfrak{u}}}_{\epsilon}^{i j}(t)\right\|_{H^{k-2}}+\left\|\epsilon \partial_{t} \overline{\mathfrak{u}}_{\epsilon}^{i j}(t)-\epsilon \partial_{t} \tilde{\overline{\mathfrak{u}}}_{\epsilon}^{i j}(t)\right\|_{H^{k-2}} \lesssim \epsilon \\
& \quad \text { for all }(t, \epsilon) \in[0, T) \times\left(0, \epsilon_{0}\right] .
\end{aligned}
$$


From the above theorem, the interpretation of the limiting solution is clear. The $\left\{\tilde{\rho}, \tilde{w}^{I}\right\}$ satisfies the standard Poisson-Euler equations of Newtonian gravity with the obvious interpretation as the fluid density and three-velocity, while the $\tilde{\mathfrak{u}}_{\epsilon}^{i j}$ represent high-frequency gravitational radiation propagating on a flat background with the fluid density and Newtonian potential acting as source terms.

\section{Reduced Einstein equations}

To aid in deriving the appropriate symmetric hyperbolic system for the gravitational variables, we temporarily introduce a new set of coordinates related to old ones by the simple rescaling

$$
\bar{x}^{J}=x^{J}, \quad \bar{x}^{4}=x^{4} / \epsilon,
$$

and let

$$
\partial_{i}=\frac{\partial}{\partial x^{i}}, \quad \bar{\partial}_{i}=\frac{\partial}{\partial \bar{x}^{i}} .
$$

In the new coordinates, the metric $\bar{g}_{i j}$ and its inverse $\bar{g}^{i j}$ are given by

$$
\left(\bar{g}_{i j}\right)=\left(\begin{array}{cc}
g_{I J} & \epsilon g_{I 4} \\
\epsilon g_{4 J} & \epsilon^{2} g_{44}
\end{array}\right) \quad \text { and } \quad\left(\bar{g}^{i j}\right)=\left(\begin{array}{cc}
g^{I J} & \epsilon^{-1} g^{I 4} \\
\epsilon^{-1} g^{4 J} & \epsilon^{-2} g^{44}
\end{array}\right) .
$$

Next, we consider the metric density

$$
\overline{\mathfrak{g}}^{i j}=\sqrt{|\bar{g}|} \bar{g}^{i j}, \quad \text { where } \quad|\bar{g}|=-\operatorname{det}\left(\bar{g}_{i j}\right) .
$$

We note that the metric $\bar{g}^{i j}$ is related to the density $\overline{\mathfrak{g}}^{i j}$ by the following formula

$$
\bar{g}^{i j}=\frac{1}{\sqrt{|\bar{g}|}} \overline{\mathfrak{g}}^{i j}, \quad \text { where } \quad|\bar{g}|=-\operatorname{det} \overline{\mathfrak{g}}^{i j}
$$

and hence

$$
\left(g^{i j}\right)=\frac{1}{\sqrt{|\bar{g}|}}\left(\begin{array}{cc}
\overline{\mathfrak{g}}^{I J} & \epsilon \overline{\mathfrak{g}}^{I 4} \\
\epsilon \overline{\mathfrak{g}}^{4 J} & \epsilon^{2} \overline{\mathfrak{g}}^{44}
\end{array}\right) .
$$

To obtain a gravitational variable that is regular and non-trivial in the limit $\epsilon \searrow 0$, we define

$$
\overline{\mathfrak{u}}^{i j}:=\frac{1}{4 \epsilon^{2}}\left(\overline{\mathfrak{g}}^{i j}-\eta^{i j}\right),
$$


where

$$
\left(\eta^{i j}\right)=\left(\begin{array}{cc}
\mathbb{I}_{3 \times 3} & 0 \\
0 & -1
\end{array}\right)
$$

is the Minkowski metric density. As stated in the introduction, for $\epsilon>0$, the metric $g_{i j}$ can be recovered from the density $\overline{\mathfrak{u}}^{i j}$ via the formulas (1.5)-(1.6). In the $\left(\bar{x}^{i}\right)$ coordinate system, the Christofell symbols are given by

$$
\bar{\Gamma}_{i j}^{k}=\epsilon^{2}\left(\overline{\mathfrak{g}}^{k m}\left(2 \overline{\mathfrak{g}}_{i \ell} \overline{\mathfrak{g}}_{j p}-\overline{\mathfrak{g}}_{i j} \overline{\mathfrak{g}}_{\ell p}\right) \bar{\partial}_{m} \overline{\overline{\mathfrak{u}}}^{\ell p}+2\left(\overline{\mathfrak{g}}_{\ell p} \delta_{(i}^{k} \bar{\partial}_{j)} \overline{\overline{\mathfrak{u}}}^{\ell p}-2 \overline{\mathfrak{g}}_{\ell(i} \bar{\partial}_{j)} \overline{\mathfrak{u}}^{k \ell}\right)\right) .
$$

These are related to the Christofell symbols in the $\left(x^{i}\right)$ coordinate system as follows

$$
\begin{gathered}
\Gamma_{44}^{A}=\epsilon^{-2} \bar{\Gamma}_{44}^{A}, \quad \Gamma_{44}^{4}=\epsilon^{-1} \bar{\Gamma}_{44}^{4}, \quad \Gamma_{A 4}^{4}=\bar{\Gamma}_{A 4}^{4}, \\
\Gamma_{A B}^{4}=\epsilon \Gamma_{A B}^{4}, \quad \Gamma_{B 4}^{A}=\epsilon^{-1} \bar{\Gamma}_{B 4}^{A} \quad \text { and } \quad \Gamma_{B C}^{A}=\bar{\Gamma}_{B C}^{A} .
\end{gathered}
$$

Using (2.6), a straightforward calculation shows that the Einstein tensor $\bar{G}^{i j}$ is given in terms of the density $\overline{\mathfrak{u}}^{i j}$ by

$$
\mathcal{G}^{i j}:=\frac{1}{2 \epsilon^{2}}|\bar{g}| \bar{G}^{i j}=\overline{\mathfrak{g}}^{k \ell} \bar{\partial}_{k \ell}^{2} \overline{\mathfrak{u}}^{i j}+\epsilon^{2}\left(A^{i j}+B^{i j}+C^{i j}\right)+D^{i j},
$$

where

$$
\begin{aligned}
|\bar{g}| & =-\operatorname{det}\left(\overline{\mathfrak{g}}^{i j}\right) \\
A^{i j} & =2\left(\frac{1}{2} \overline{\mathfrak{g}}_{k \ell} \overline{\mathfrak{g}}_{m n}-\overline{\mathfrak{g}}_{k m} \overline{\mathfrak{g}}_{\ell n}\right)\left(\overline{\mathfrak{g}}^{i p} \overline{\mathfrak{g}}^{j q}-\frac{1}{2} \overline{\mathfrak{g}}^{i j} \overline{\mathfrak{g}}^{p q}\right) \bar{\partial}_{p} \overline{\mathfrak{u}}^{k \ell} \bar{\partial}_{q} \overline{\mathfrak{u}}^{m n} \\
B^{i j} & =4 \overline{\mathfrak{g}}_{k \ell}\left(2 \overline{\mathfrak{g}}^{n(i} \bar{\partial}_{m} \overline{\overline{\mathfrak{u}}}^{j) \ell} \bar{\partial}_{n} \overline{\mathfrak{u}}^{k m}-\frac{1}{2} \overline{\mathfrak{g}}^{i j} \bar{\partial}_{m} \overline{\mathfrak{u}}^{k n} \bar{\partial}_{n} \overline{\mathfrak{u}}^{m \ell}-\overline{\mathfrak{g}}^{m n} \bar{\partial}_{m} \overline{\overline{\mathfrak{u}}}^{i k} \bar{\partial}_{n} \overline{\mathfrak{u}}^{j \ell}\right) \\
C^{i j} & =4\left(\bar{\partial}_{k} \overline{\overline{\mathfrak{u}}}^{i j} \bar{\partial}_{\ell} \overline{\overline{\mathfrak{u}}}^{k \ell}-\bar{\partial}_{k} \overline{\mathfrak{u}}^{i \ell} \bar{\partial}_{\ell} \overline{\mathfrak{u}}^{j k}\right) \\
D^{i j} & =\overline{\mathfrak{g}}^{i j} \bar{\partial}_{k \ell}^{2} \overline{\mathfrak{u}}^{k \ell}-2 \bar{\partial}_{k \ell}^{2} \overline{\mathfrak{u}}^{k(i} \overline{\mathfrak{g}}^{j \ell \ell}
\end{aligned}
$$

To fix the gauge, we assume that

$$
\bar{\partial}_{i} \overline{\mathfrak{u}}^{i j}=0 .
$$

For $\epsilon>0$, this is equivalent to to the harmonic gauge

$$
\partial_{i} \mathfrak{g}^{i j}=\partial_{i}\left(\sqrt{-\operatorname{det}\left(g_{k \ell}\right)} g^{i j}\right)=0 .
$$


Setting

$$
\mathcal{G}_{R}^{i j}:=\mathcal{G}^{i j}-D^{i j}=\overline{\mathfrak{g}}^{k \ell} \bar{\partial}_{k \ell}^{2} \overline{\mathfrak{u}}^{i j}+\epsilon^{2}\left(A^{i j}+B^{i j}+C^{i j}\right)
$$

and

$$
\mathcal{T}^{i j}:=\epsilon^{2}|\bar{g}| \bar{T}^{i j}=|\bar{g}|\left(\begin{array}{cc}
\epsilon^{2} T^{I J} & \epsilon^{1} T^{I 4} \\
\epsilon^{1} T^{4 J} & T^{44}
\end{array}\right)
$$

the Einstein equations $G^{i j}=2 \epsilon^{4} T^{i j}$ in the gauge (2.15) become

$$
\mathcal{G}_{R}^{i j}=\mathcal{T}^{i j}
$$

We will refer to these as the reduced Einstein equations.

To write the reduced Einstein equations in first order form, we introduce the variables

$$
\overline{\mathfrak{u}}_{k}^{i j}:=\bar{\partial}_{k} \overline{\mathfrak{u}}^{i j}= \begin{cases}\partial_{I} \overline{\mathfrak{u}}^{i j} & \text { if } k=I, \\ \epsilon \partial_{4} \overline{\mathfrak{u}}^{i j} & \text { if } k=4 .\end{cases}
$$

The reduced Einstein equations then become

$$
\begin{aligned}
-\overline{\mathfrak{g}}^{44} \bar{\partial}_{4} \overline{\mathfrak{u}}_{4}^{i j} & =2 \overline{\mathfrak{g}}^{4 I} \bar{\partial}_{I} \overline{\mathfrak{u}}_{4}^{i j}+\overline{\mathfrak{g}}^{I J} \bar{\partial}_{I} \overline{\overline{\mathfrak{u}}}_{J}^{i j}+\epsilon^{2}\left(A^{i j}+B^{i j}+C^{i j}\right)-\mathcal{T}^{i j}, \\
\overline{\mathfrak{g}}^{I J} \bar{\partial}_{4} \overline{\mathfrak{u}}_{J}^{i j} & =\overline{\mathfrak{g}}^{I J} \bar{\partial}_{J} \overline{\mathfrak{u}}_{4}^{i j}, \\
\bar{\partial}_{4} \overline{\mathfrak{u}}^{i j} & =\overline{\mathfrak{u}}_{4}^{i j},
\end{aligned}
$$

or equivalently

$$
\begin{aligned}
-\overline{\mathfrak{g}}^{44} \partial_{4} \overline{\mathfrak{u}}_{4}^{i j} & =\frac{2}{\epsilon} \overline{\mathfrak{g}}^{4 I} \partial_{I} \overline{\mathfrak{u}}_{4}^{i j}+\frac{1}{\epsilon} \overline{\mathfrak{g}}^{I J} \partial_{I} \overline{\mathfrak{u}}_{J}^{i j}+\epsilon\left(A^{i j}+B^{i j}+C^{i j}\right)-\frac{1}{\epsilon} \mathcal{T}^{i j}, \\
\overline{\mathfrak{g}}^{I J} \partial_{4} \overline{\mathfrak{u}}_{J}^{i j} & =\frac{1}{\epsilon} \overline{\mathfrak{g}}^{I J} \partial_{J} \overline{\mathfrak{u}}_{4}^{i j}, \\
\partial_{4} \overline{\mathfrak{u}}^{i j} & =\frac{1}{\epsilon} \overline{\mathfrak{u}}_{4}^{i j} .
\end{aligned}
$$

Next, we define

$$
\mathfrak{u}^{i j}=\epsilon \overline{\mathfrak{u}}^{i j}, \quad \mathfrak{u}_{k}^{i j}=\overline{\mathfrak{u}}_{k}^{i j}
$$

and let

$$
\mathcal{V}=\left\{\left(r^{i j}\right) \in \mathbb{M}_{4 \times 4} \mid \operatorname{det}\left(\eta^{i j}+4 r^{i j}\right)>0\right\}
$$


Then using vector notation

$$
\mathfrak{u}^{i j}=\left(\mathfrak{u}_{4}^{i j}, \mathfrak{u}_{J}^{i j}, \mathfrak{u}^{i j}\right)^{\mathrm{T}}
$$

the reduced Einstein equations take the form

$$
A^{4}(\epsilon \mathfrak{u}) \partial_{4} \mathfrak{u}^{i j}=\frac{1}{\epsilon} C^{I} \partial_{I} \mathfrak{u}^{i j}+A^{I}(\mathfrak{u}) \partial_{I} \mathfrak{u}^{i j}+\bar{F}^{i j}(\epsilon, \mathfrak{u})-\frac{1}{\epsilon}\left(\mathcal{T}^{i j}, 0,0\right)^{\mathrm{T}}
$$

where

$$
\begin{gathered}
A^{4}(\epsilon \mathfrak{u})=\left(\begin{array}{ccc}
1-4 \epsilon \mathfrak{u}^{44} & 0 & 0 \\
0 & \delta^{I J}+4 \epsilon \mathfrak{u}^{I J} & 0 \\
0 & 0 & 1
\end{array}\right), \\
C^{I}=\left(\begin{array}{ccc}
0 & \delta^{I J} & 0 \\
\delta^{I J} & 0 & 0 \\
0 & 0 & 0
\end{array}\right) \\
A^{I}(\mathfrak{u})=\left(\begin{array}{ccc}
8 \mathfrak{u}^{4 I} & 4 \mathfrak{u}^{I J} & 0 \\
4 \mathfrak{u}^{I J} & 0 & 0 \\
0 & 0 & 0
\end{array}\right) \\
\bar{F}_{0}^{i j}(\mathfrak{u})=\left(0,0, \mathfrak{u}_{4}^{i j}\right)^{\mathrm{T}}
\end{gathered}
$$

and

$$
\bar{F}^{i j}(\overline{\mathfrak{u}}, \epsilon \mathfrak{u})=\left(A^{i j}+B^{i j}+C^{i j}, 0,0\right)^{\mathrm{T}}
$$

Here, we are using the notation

$$
\mathfrak{u}=\left(\mathfrak{u}^{i j}\right) \quad \text { and } \quad \mathfrak{u}_{k}=\left(\mathfrak{u}_{k}^{i j}\right)
$$

The stress-energy tensor is given in terms of the $\mathfrak{u}$ variable by

$$
\begin{aligned}
\left(T^{i j}\right)= & \rho\left(v^{i} v^{j}\right)+\frac{1}{\sqrt{|\bar{g}|}}\left(\begin{array}{cc}
\delta^{I J} p & 0 \\
0 & 0
\end{array}\right)+\frac{\epsilon}{\sqrt{|\bar{g}|}}\left(\begin{array}{cc}
4 \mathfrak{u}^{I J} p & 0 \\
0 & 0
\end{array}\right) \\
& +\epsilon^{2}\left(p\left(v^{i} v^{j}\right)+\frac{p}{\sqrt{|\bar{g}|}}\left(\begin{array}{cc}
0 & 4 \mathfrak{u}^{I 4} \\
4 \mathfrak{u}^{4 J} & -1+4 \epsilon \mathfrak{u}^{44}
\end{array}\right)\right)
\end{aligned}
$$

which we can write as

$$
\frac{1}{\epsilon}\left(\mathcal{T}^{i j}\right)=\left(\begin{array}{cc}
0 & 0 \\
0 & \epsilon^{-1} \rho
\end{array}\right)+\mathcal{S}^{i j}
$$


where

$$
\begin{aligned}
\left(\mathcal{S}^{i j}\right)= & \rho\left(\begin{array}{cc}
0 & |\bar{g}| v^{I} v^{4} \\
|\bar{g}| v^{J} v^{4} & \epsilon^{-1}\left[(|\bar{g}|-1)\left(v^{4}\right)^{2}+\left(\left(v^{4}\right)^{2}-1\right)\right]
\end{array}\right) \\
& +\epsilon|\bar{g}|\left(\begin{array}{cc}
\left(\rho+\epsilon^{2} p\right) v^{I} v^{J}+|\bar{g}|^{-1 / 2} p\left(\delta^{I J}+4 \epsilon \mathfrak{u}^{I J}\right) & \epsilon p v^{I} v^{4}+4 \epsilon|\bar{g}|^{-1 / 2} p \mathfrak{u}^{I 4} \\
\epsilon p v^{J} v^{4}+4 \epsilon|\bar{g}|^{-1 / 2} p \mathfrak{u}^{4 J} & p\left(v^{4}\right)^{2}+|\bar{g}|^{-1 / 2} p\left(-1+4 \epsilon \mathfrak{u}^{44}\right)
\end{array}\right) .
\end{aligned}
$$

Letting (see (1.7))

$$
\mathbf{w}=\left(\alpha, w^{i}\right)^{\mathrm{T}},
$$

we can decompose $\mathcal{S}^{i j}$ as

$$
\mathcal{S}^{i j}=\mathcal{S}_{0}^{i j}+\epsilon \mathcal{S}_{1}^{i j},
$$

where

$$
\begin{aligned}
\mathcal{S}_{0}^{i j} & (\mathfrak{u}, \mathbf{w}, \epsilon \mathfrak{u}, \epsilon \mathbf{w}) \\
& =\rho\left(\begin{array}{cc}
0 & |\bar{g}| w^{I}\left(1+\epsilon w^{4}\right) \\
|\bar{g}| w^{J}\left(1+\epsilon w^{4}\right) & \epsilon^{-1}\left[(|\bar{g}|-1)\left(1+\epsilon w^{4}\right)^{2}+\left(\left(1+\epsilon w^{4}\right)^{2}-1\right)\right]
\end{array}\right),
\end{aligned}
$$

and

$$
\begin{aligned}
& \mathcal{S}_{1}^{i j}(\mathbf{w}, \epsilon \mathfrak{u}, \epsilon \mathbf{w}) \\
& \quad=|\bar{g}|\left(\begin{array}{c}
\rho w^{I} w^{J}+p \epsilon w^{I} \epsilon w^{J}+|\bar{g}|^{-1 / 2} p \overline{\mathfrak{g}}^{I J} p \epsilon w^{J}\left(1+\epsilon w^{4}\right)+4|\bar{g}|^{-1 / 2} p \epsilon \mathfrak{u}^{J 4} \\
p \epsilon w^{I}\left(1+\epsilon w^{4}\right)+4|\bar{g}|^{-1 / 2} p \epsilon u^{I 4} p\left(1+\epsilon w^{4}\right)^{2}+|\bar{g}|^{-1 / 2} p\left(-1+4 \epsilon u^{44}\right)
\end{array}\right) .
\end{aligned}
$$

\section{$3 \quad$ Regularized Euler equations}

In the coordinates $\left(\bar{x}^{i}\right)$, the Euler equations are given by

$$
\bar{\nabla}_{i} \bar{T}^{i j}=0,
$$

where $\bar{T}^{i j}=\left(\rho+\epsilon^{2} p\right) \bar{v}^{i} \bar{v}^{j}+p \bar{g}^{i j}$ and the fluid velocity $\bar{v}^{i}$ is normalized according to

$$
\bar{v}_{i} \bar{v}^{i}=-\frac{1}{\epsilon^{2}} .
$$


To write (3.1) as a symmetric hyperbolic system, we follow [2] and differentiate (3.2) to get

$$
\bar{v}_{i} \bar{\nabla}_{j} \bar{v}^{i}=0 \quad \text { and } \quad \bar{v}^{j} \bar{v}_{i} \bar{\nabla}_{j} \bar{v}^{i}=0 .
$$

Writing out (3.1) explicitly, we have

$$
\left(\bar{\partial}_{i} \rho+\epsilon^{2} \bar{\partial}_{i} p\right) \bar{v}^{i} \bar{v}^{j}+\left(\rho+\epsilon^{2} p\right)\left(\bar{v}^{j} \bar{\nabla}_{i} \bar{v}^{i}+\bar{v}^{i} \bar{\nabla}_{i} \bar{v}^{j}\right)+\bar{g}^{i j} \bar{\partial}_{i} p=0 .
$$

The operator

$$
L_{i}^{j}=\delta_{i}^{j}+\epsilon^{2} \bar{v}^{j} \bar{v}_{i}
$$

projects into the subspace orthogonal to the fluid velocity $\bar{v}^{i}$, i.e., $L_{i}^{j} L_{k}^{i}=L_{k}^{j}$ and $L_{i}^{j} \bar{v}^{i}=0$. Using $L_{k}^{j}$ to project the Euler (3.4) into components parallel and orthogonal to $\bar{v}^{i}$ yields, after using the relations (3.2) to (3.3), the following system:

$$
\begin{gathered}
\bar{v}^{i} \bar{\partial}_{i} \rho+\left(\rho+\epsilon^{2} p\right) L_{j}^{i} \bar{\nabla}_{i} \bar{v}^{j}=0, \\
M_{i j} \bar{v}^{k} \bar{\nabla}_{k} \bar{v}^{j}+\frac{1}{\rho+\epsilon^{2} p} L_{j}^{i} \bar{\partial}_{i} p=0,
\end{gathered}
$$

where

$$
M_{i j}=\bar{g}_{i j}+2 \epsilon^{2} \bar{v}_{i} \bar{v}_{j}
$$

As discussed in the introduction, we use a Makino density variable $\alpha$ (see (1.9)) to regularize the fluid equations in regions where the density and pressure vanish. After multiplying (3.5) by the square of the function

$$
h(\epsilon \alpha)=\left(1+\frac{1}{4 n(n+1)}(\epsilon \alpha)^{2}\right),
$$

a short calculation shows that the Makino density $\alpha$ and the fluid fourvelocity $\bar{v}^{i}$ satisfy

$$
\begin{gathered}
h^{2} \bar{v}^{i} \bar{\partial}_{i} \alpha+q L_{j}^{i} \bar{\nabla}_{i} \bar{v}^{j}=0, \\
M_{i j} \bar{v}^{k} \bar{\nabla}_{k} \bar{v}^{j}+q L_{i}^{j} \bar{\partial}_{j} \alpha=0,
\end{gathered}
$$

where

$$
s^{2}=\frac{d p}{d \rho}=\frac{1}{4 n^{2}} \alpha^{2}
$$


is the square of the speed of sound, and

$$
q=\frac{1}{2 n h(\epsilon \alpha)} \alpha
$$

Instead of solving (3.7) to (3.8), we consider the following modified system

$$
\begin{gathered}
h^{2} \bar{v}^{i} \bar{\partial}_{i} \alpha+q L_{j}^{i} \bar{\nabla}_{i} \bar{v}^{j}=0, \\
M_{i j} \bar{v}^{k} \bar{\nabla}_{k} \bar{v}^{j}+q L_{i}^{j} \bar{\partial}_{j} \alpha+\left(\chi_{4 \bar{R}}-1\right) M_{i j} \bar{\Gamma}_{k \ell}^{j} \bar{v}^{k} \bar{v}^{\ell}=0,
\end{gathered}
$$

Here we are using

$$
\chi_{\lambda}(x):=\chi(x / \lambda) \quad \lambda>0,
$$

where $\chi \in C^{\infty}\left(\mathbb{R}^{3}\right)$ is a smooth cutoff function satisfying $\chi(x)=1$ for $|x| \leq$ $1, \chi(x)=0$ for $|x| \geq 2$, and $0 \leq \chi(x) \leq 1$ for all $x \in \mathbb{R}^{3}$.

Since $w^{I}=\bar{v}^{I}$ and $w^{4}=\bar{v}^{4}-1 / \epsilon$, we can write (3.9) and (3.10) as

$$
a^{4} \partial_{4} \mathbf{w}=a^{I} \partial_{I} \mathbf{w}+b
$$

where

$$
\begin{aligned}
& a^{4}=\left(\begin{array}{cc}
h^{2}\left(1+\epsilon w^{4}\right) & \epsilon q L_{j}^{4} \\
\epsilon q L_{i}^{4} & M_{i j}\left(1+\epsilon w^{4}\right)
\end{array}\right), \\
& a^{I}=\left(\begin{array}{cc}
-h^{2} w^{I} & -q L_{j}^{I} \\
-q L_{i}^{I} & -M_{i j} w^{I}
\end{array}\right)
\end{aligned}
$$

and

$$
b=\left(\begin{array}{c}
-q L_{j}^{i} \bar{\Gamma}_{i \ell}^{j} \bar{v}^{\ell} \\
-\chi_{4} \bar{R}_{i j} \bar{\Gamma}_{k \ell}^{j} \bar{v}^{k} \bar{v}^{\ell}
\end{array}\right) .
$$

From (2.3), (2.5), (2.19), and (2.30), we find that

$$
\bar{g}_{i j}=\eta_{i j}+f_{i j}(\epsilon \mathfrak{u})
$$


where the $f_{i j}(y)$ are analytic and satisfy $f_{i j}(y)=\mathrm{O}(|y|)$ as $y \rightarrow 0$, while (2.6) shows that

$$
\bar{\Gamma}_{i j}^{k}=\epsilon\left[\eta^{k m}\left(2 \eta_{i \ell} \eta_{j p}-\eta_{i j} \eta_{\ell p}\right) \epsilon \mathfrak{u}_{m}^{l p}+2\left(\eta_{\ell p} \delta_{(i}^{k} \epsilon \mathfrak{u}_{j)}^{\ell p}-2 \eta_{\ell(i} \in \mathfrak{u}_{j}^{k \ell}\right)\right]+\epsilon f_{i j}^{k}\left(\epsilon \mathfrak{u}, \epsilon \mathfrak{u}_{m}\right)
$$

for functions $f_{i j}^{k}\left(\epsilon \mathfrak{u}, \epsilon \mathfrak{u}_{m}\right)$ that are analytic for $\epsilon \mathfrak{U} \in \mathcal{V}$, linear in the $\epsilon \mathfrak{u}_{m}$, and satisfy $f_{i j}^{k}(0, y)=0$. The expansion (3.15) allows us to write

$$
M_{i j}=\bar{g}_{i j}+2 \epsilon^{2} \bar{g}_{i k} \bar{g}_{j \ell} \bar{v}^{k} \bar{v}^{\ell}=\delta_{i j}+m_{i j}\left(\epsilon \mathfrak{u}, \epsilon w^{k}\right),
$$

and

$$
L_{i}^{j}=\delta_{i}^{j}+\epsilon^{2} \bar{g}_{i k} \bar{v}^{k} \bar{v}^{j}=\delta_{i}^{j}-\delta_{i}^{4} \delta_{4}^{j}+\ell_{i}^{j}\left(\epsilon \mathfrak{u}, \epsilon w^{k}\right)
$$

for functions $\ell_{i}^{j}\left(\epsilon \mathfrak{u}, \epsilon w^{k}\right)$ and $m_{i j}\left(\epsilon \mathfrak{U}, \epsilon w^{k}\right)$ that satisfy $\ell_{i}^{j}(0,0)=m_{i j}(0,0)=$ 0 , and are analytic for $\epsilon \mathfrak{u} \in \mathcal{V}$. Using (3.15) to (3.18), we can express the $a^{i}$ and $b$ as

$$
\begin{aligned}
a^{4} & =\left(\begin{array}{cc}
1 & 0 \\
0 & \delta_{i j}
\end{array}\right)+\hat{a}^{4}(\epsilon \mathfrak{u}, \epsilon \mathbf{w}), \\
a^{I} & =\left(\begin{array}{cc}
-w^{I} & -\frac{\alpha}{2 n} \delta_{j}^{I} \\
-\frac{\alpha}{2 n} \delta_{i}^{I} & -\delta_{i j} w^{I}
\end{array}\right)+w^{I} \hat{a}(\epsilon \mathfrak{u}, \epsilon \mathbf{w})+\alpha \hat{a}^{I}(\epsilon \mathfrak{u}, \epsilon \mathbf{w}),
\end{aligned}
$$

and

$$
\begin{aligned}
b= & \left(\begin{array}{c}
0 \\
\chi_{4 \bar{R}}\left[-\eta^{i m}\left(2 \eta_{4 \ell} \eta_{4 p}+\eta_{\ell p}\right) \mathfrak{u}_{m}^{l p}-2\left(\eta_{\ell p} \delta_{4}^{i} \mathfrak{u}_{4}^{\ell p}-2 \eta_{\ell 4} \mathfrak{u}_{4}^{i \ell}\right)\right]
\end{array}\right) \\
& +\left(\begin{array}{c}
\alpha \hat{b}_{1}(\epsilon \mathfrak{u}, \epsilon \mathbf{w}) \cdot \epsilon \mathfrak{u}_{k} \\
\chi_{4 \bar{R}} \hat{b}_{2}(\epsilon \mathfrak{u}, \epsilon \mathbf{W}) \cdot \mathfrak{u}_{k}
\end{array}\right) .
\end{aligned}
$$

We observe that the matrices $\hat{a}^{4}, \hat{a}$, and $\hat{a}^{I}$ are symmetric, and the maps $\hat{a}^{4}$, $\hat{a}, \hat{a}^{I}, \hat{b}_{1}$, and $\hat{b}_{2}$ are analytic (for $\epsilon \mathfrak{u} \in \mathcal{V}$ ) and satisfy $\hat{a}^{4}(0,0)=0, \hat{a}^{I}(0,0)=$ $0, \hat{a}(0,0)=0, \hat{b}_{1}(0,0)=0$, and $\hat{b}_{2}(0,0)=0$. This shows that the system (3.11) is symmetric hyperbolic on a region where $(\epsilon \mathfrak{u}, \epsilon \mathbf{w})$ is small enough to ensure that $a^{4}$ is positive definite. This can always be arranged by taking $\epsilon$ small enough and since we are interested in the limit $\epsilon \searrow 0$ no generality is lost by assuming this. 


\section{Uniform local existence}

The combined systems (2.26) and (3.11) can be written as

$$
\begin{aligned}
b^{0}\left(\epsilon V, \epsilon^{2} U\right) \partial_{t} V= & \frac{1}{\epsilon} c^{I} \partial_{I} V+b^{I}\left(V, \epsilon U, \epsilon V, \epsilon^{2} U\right) \partial_{I} V \\
& +f_{0}\left(V, \epsilon U, \epsilon V, \epsilon^{2} U\right)+\epsilon f_{1}\left(V, \epsilon U, \epsilon V, \epsilon^{2} U\right)+\frac{1}{\epsilon} g(V),
\end{aligned}
$$

where

$$
\begin{aligned}
& U=\left(0,0, \overline{\mathfrak{u}}_{o}^{i j}, 0,0\right)^{\mathrm{T}}, \quad \overline{\mathfrak{u}}_{o}^{i j}=\left.\overline{\mathfrak{u}}^{i j}\right|_{t=0}, \\
& V=\left(\mathfrak{u}_{4}^{i j}, \mathfrak{u}_{J}^{i j}, \delta \mathfrak{u}^{i j}, \alpha, w^{i}\right)^{\mathrm{T}}, \quad \delta \mathfrak{u}^{i j}=\mathfrak{u}^{i j}-\epsilon \overline{\mathfrak{u}}_{o}^{i j}, \\
& b^{0}\left(\epsilon V, \epsilon^{2} U\right)=\left(\begin{array}{cc}
A^{4}(\epsilon \mathfrak{u}) & 0 \\
0 & a^{4}(\epsilon \mathfrak{u}, \epsilon \mathbf{W})
\end{array}\right), \\
& c^{I}=\left(\begin{array}{cc}
C^{I} & 0 \\
0 & 0
\end{array}\right) \text {, } \\
& b^{I}\left(V, \epsilon U, \epsilon V, \epsilon^{2} U\right)=\left(\begin{array}{cc}
A^{I}(\mathfrak{u}) & 0 \\
0 & a^{I}(\mathbf{w}, \epsilon \mathfrak{u}, \epsilon \mathbf{w})
\end{array}\right), \\
& f_{0}\left(V, \epsilon U, \epsilon V, \epsilon^{2} U\right)=\left(\begin{array}{c}
\bar{F}_{0}^{i j}(\mathfrak{u})-\mathcal{S}_{0}^{i j}(\mathfrak{u}, \mathbf{w}, \epsilon \mathfrak{u}, \epsilon \mathbf{w}) \\
b(\mathfrak{u}, \mathbf{w}, \epsilon \mathfrak{u}, \epsilon \mathbf{w})
\end{array}\right), \\
& f_{1}\left(V, \epsilon U, \epsilon V, \epsilon^{2} U\right)=\left(\begin{array}{c}
\bar{F}_{1}^{i j}(\mathfrak{u}, \epsilon \mathfrak{u})-\mathcal{S}_{1}^{i j}(\mathbf{w}, \epsilon \mathfrak{u}, \epsilon \mathbf{w}) \\
0
\end{array}\right),
\end{aligned}
$$

and

$$
g(V)=\left(-\delta_{4}^{i} \delta_{4}^{j} \rho(\alpha), 0, \ldots, 0\right)^{\mathrm{T}} .
$$

For initial data, we will often use the notation

$$
\underset{o}{z}=\left.z\right|_{t=0}
$$

In addition to solving these evolution equations, we must also solve the following constraint equations on the initial hypersurface 
$\Sigma=\left\{\left(x^{I}, 0\right) \mid\left(x^{I}\right) \in \mathbb{R}^{3}\right\}$ to get a full solution to the Einstein-Euler equations:

$$
\begin{aligned}
\mathcal{C}^{j} & :=\mathcal{G}^{4 i}-\mathcal{T}^{4 i}=0 \quad \text { (gravitational constraint equations) } \\
\mathcal{H}^{j} & :=\bar{\partial}_{i} \overline{\mathfrak{u}}^{i j}=0 \quad \text { (harmonic gauge condition) }
\end{aligned}
$$

and

$$
\mathcal{N}:=\epsilon \bar{v}_{i} \bar{v}^{i}+\frac{1}{\epsilon}=0 \quad \text { (fluid velocity normalization) }
$$

To fix a region on which the system where both the evolution (4.1) and constraint (4.10) to (4.12) are well defined, we note from (2.20), (3.19), and the invertibility of the Lorentz metric $\left(\eta^{i j}\right)$ that there exists a constant $K_{0}>0$ such that

$$
\begin{gathered}
-\operatorname{det}\left(\eta^{i j}+4 \epsilon \mathfrak{u}^{i j}\right)>1 / 16, \quad 1+\epsilon w^{4}>1 / 16, \\
A^{4}(\epsilon \mathfrak{u}) \geq \frac{1}{16} \mathbb{I}, \quad a^{4}(\epsilon \mathfrak{u}, \epsilon \mathbf{w}) \geq \frac{1}{16} \mathbb{I},
\end{gathered}
$$

and

$$
\left|A^{4}(\epsilon \mathfrak{u})\right| \leq 16, \quad\left|a^{4}(\epsilon \mathfrak{u}, \epsilon \mathbf{W})\right| \leq 16
$$

for all $|\epsilon \mathfrak{u}| \leq 2 K_{0},\left|\epsilon w^{i}\right| \leq 2 K_{0},|\epsilon \alpha| \leq 2 K_{0}$. The choice of the bounds $1 / 16$ and 16 is somewhat arbitrary, and they can be replaced by any number of the form $1 / M$ and $M$ for any $M>1$ without changing any of the arguments presented in the following sections. However, since we are interested in the limit $\epsilon \searrow 0$, we lose nothing by assuming $M=16$.

\subsection{Newtonian initial data}

To generate a one parameter family of solutions to the constraint (4.10) to (4.12) that is regular in the limit $\epsilon \searrow 0$, we use a slight variation of the method used in [10], which is based on previous work by Lottermoser [8]. Before we state the theorem, we note from (1.9), (1.8), and the weighted multiplication inequality (see [10] Lemma A.8) that if $\alpha \in H_{\delta}^{k}(\delta \leq 0, k>$ 3/2) then $\rho, p \in H_{\delta}^{k}$.

Proposition 4.1. Suppose $-1<\delta<0, k>3 / 2+1, R>0$ and $\left(\tilde{\rho}, \tilde{p}, \tilde{w}^{I}\right.$, $\left.\tilde{\mathfrak{z}}_{4}^{I J}, \tilde{\mathfrak{z}}^{I J}\right) \in\left(H_{\delta-2}^{k-2}\right)^{2} \times H_{\delta-1}^{k} \times H_{\delta-1}^{k-1} \times B_{R}\left(H_{\delta}^{k}\right)$. Then there exists an $\epsilon_{0}>0$, 
an open neighborhood $U$ of $\left(\tilde{\rho}, \tilde{p}, \tilde{w}^{I}, \tilde{\mathfrak{z}}_{4}^{I J}, \tilde{\mathfrak{z}}^{I J}\right)$, and analytic maps $\left(-\epsilon_{0}, \epsilon_{0}\right) \times$ $U \rightarrow H_{\delta-1}^{k}:\left(\epsilon, \rho, p, w^{I}, \mathfrak{z}_{4}^{I J}, \mathfrak{z}^{I J}\right) \mapsto w^{4},\left(-\epsilon_{0}, \epsilon_{0}\right) \times U \rightarrow H_{\delta}^{k}:\left(\epsilon, \rho, p, w^{I}, \mathfrak{z}_{4}^{I J}\right.$, $\left.\mathfrak{z}^{I J}\right) \mapsto \phi,\left(-\epsilon_{0}, \epsilon_{0}\right) \times U \rightarrow H_{\delta}^{k}:\left(\epsilon, \rho, p, w^{I}, \mathfrak{z}_{4}^{I J}, \mathfrak{z}^{I J}\right) \mapsto \mathfrak{w}^{I}$ such that for each $\left(\rho, p, w^{I}, \mathfrak{z}_{4}^{I J}, \mathfrak{z}^{I J}\right) \in U,\left(\epsilon, \rho, p, w^{I}, w^{4}, \overline{\mathfrak{u}}_{4}^{i j}, \bar{\partial}_{4} \overline{\mathfrak{u}}^{i j}\right)$ is a solution to the three constraints

$$
\mathcal{C}^{j}=0, \quad \mathcal{H}^{j}=0, \quad \text { and } \quad \mathcal{N}=0
$$

where

$$
\begin{aligned}
\left(\overline{\mathfrak{u}}^{i j}\right) & =\left(\begin{array}{cc}
\mathfrak{z}^{I J} & \mathfrak{w}^{I} \\
\mathfrak{w}^{J} & \phi
\end{array}\right) \\
\left(\epsilon \partial_{t} \overline{\mathfrak{u}}^{i j}\right) & =\left(\begin{array}{cc}
\mathfrak{z}_{4}^{I J} & -\partial_{K} \mathfrak{z}^{K I} \\
-\partial_{K} \mathfrak{z}^{K J} & -\partial_{K} \mathfrak{w}^{K}
\end{array}\right),
\end{aligned}
$$

and

$$
w^{4}=-\frac{1}{\epsilon}+\frac{-\epsilon \bar{g}_{4 J} w^{J}-\sqrt{\epsilon^{2}\left(\bar{g}_{4 J} w^{J}\right)^{2}-\bar{g}_{44}\left(\epsilon^{2} \bar{g}_{I J} w^{I} w^{J}+1\right)}}{\epsilon \bar{g}_{44}} .
$$

Moreover, if we let $\phi_{0}=\left.\phi\right|_{\epsilon=0}, \mathfrak{w}_{0}^{I}=\left.\mathfrak{w}^{I}\right|_{\epsilon=0}$, and $w_{0}^{4}=\left.w^{4}\right|_{\epsilon=0}$, then $\phi_{0}, \mathfrak{w}_{0}^{I}$, and $w_{0}^{4}$ satisfy the equations

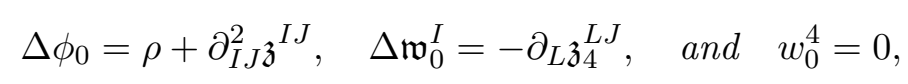

respectively.

Proof. The proof follows from a simple adaptation of the proof of Proposition 5.1 in $[10]$.

Corollary 4.2. For $-1<\delta<-1 / 2$, the ADM mass of the 1-parameter family of initial data constructed in Proposition 4.1 satisfies

$$
m_{\mathrm{ADM}}=\int_{\mathbb{R}^{3}} \rho d x^{3}+\mathrm{O}(\epsilon)
$$

Proof. For fixed $\left(\rho, p, w^{I}, \mathfrak{z}_{4}^{I J}, \mathfrak{z}^{I J}\right) \in\left(H_{\delta-2}^{k-2}\right)^{2} \times H_{\delta-1}^{k} \times H_{\delta-1}^{k-1} \times H_{\delta}^{k}$, it follows from Proposition 4.1 that for $\epsilon_{0}$ small enough, the maps

$$
\left[0, \epsilon_{0}\right) \ni \epsilon \longmapsto \bar{g}_{i j} \in H_{\delta}^{k} \quad \text { and } \quad\left[0, \epsilon_{0}\right) \ni \epsilon \longmapsto \epsilon \partial_{t} \bar{g}_{i j} \in H_{\delta-1}^{k-1}
$$


are analytic (see (2.1) and (2.5)). Moreover, a short calculation shows that

$$
\bar{g}_{i j}^{\epsilon}=\eta_{i j}+2 \epsilon^{2}\left(\eta_{k l} u^{k \ell} \eta_{i j}-2 \eta_{i k} u^{k \ell} \eta_{\ell j}\right)+\mathrm{O}\left(\epsilon^{3}\right),
$$

and

$$
\epsilon \partial_{t} \bar{g}_{i j}=2 \epsilon^{2}\left(\eta_{k l} u_{4}^{k \ell} \eta_{i j}-2 \eta_{i k} u_{4}^{k \ell} \eta_{\ell j}\right)+\mathrm{O}\left(\epsilon^{3}\right)
$$

where

$$
\begin{aligned}
& \left(u^{i j}\right)=\left(\begin{array}{ll}
\mathfrak{z}^{I J} & \mathfrak{w}_{0}^{I} \\
\mathfrak{w}_{0}^{J} & \phi_{0}
\end{array}\right), \\
& \left(u_{4}^{i j}\right)=\left(\begin{array}{cc}
\mathfrak{z}_{4}^{I J} & -\partial_{K} \mathfrak{z}^{K I} \\
-\partial_{K \mathfrak{z}}^{K J} & -\partial_{K} \mathfrak{w}_{0}^{K}
\end{array}\right),
\end{aligned}
$$

and

$$
\Delta \phi_{0}=\rho+\partial_{I J \mathfrak{z}}^{2} \mathfrak{z}^{I J}, \quad \Delta \mathfrak{w}_{0}^{I}=-\partial_{L \mathfrak{z}_{4}^{L J}} .
$$

Since $-1<\delta<-1 / 2$, it follows from Proposition 4.5 of [1] that the total ADM energy-momentum $\mathbb{P}=\left(\mathbb{P}_{j}\right)$ for the initial data $\left(\bar{g}_{i j}, \epsilon \partial_{t} \bar{g}_{i j}\right)$ on the initial hypersurface $\Sigma=\left\{\left(x^{I}, 0\right) \mid\left(x^{I}\right) \in \mathbb{R}^{3}\right\}$ can be calculated using the standard formulas

$$
\begin{aligned}
& \mathbb{P}_{4}=-\frac{1}{4} \oint_{S_{\infty}}\left(\partial^{I} \bar{g}_{I J}-\delta^{I K} \partial_{J} \bar{g}_{I K}\right) d S^{J}, \\
& \mathbb{P}_{I}=\frac{1}{2} \oint_{S_{\infty}}\left(\bar{K}_{K}^{K} \delta_{I J}-K_{I J}\right) d S^{J},
\end{aligned}
$$

where the extrinsic curvature $\bar{K}_{I J}$ is given by (see (2.6))

$$
\bar{K}_{I J}=-\sqrt{\frac{-1}{\bar{g}^{44}}} \bar{\Gamma}_{I J}^{4}
$$

Furthermore, the map

$$
\left[0, \epsilon_{0}\right) \ni \epsilon \longmapsto\left(\mathbb{P}_{j}\right) \in \mathbb{R}^{4}
$$

is smooth by (4.16) and Theorem 5.1 of [1]. 
By (2.6), (4.17), (4.18), and (4.24), we find the following expansions for the extrinsic curvature:

$$
\bar{K}_{I J}=\epsilon^{2}\left(2 u_{4}^{I J}+\delta_{I J}\left(u_{4}^{44}-\delta_{k \ell} u_{4}^{k \ell}\right)+2 \partial_{J} u^{4 I}+2 \partial_{I} u^{4 J}\right)+\mathrm{O}\left(\epsilon^{3}\right) .
$$

The smoothness of the map (4.25) and the two expansions (4.17) and (4.26) show that the ADM energy-momentum can be expanded as

$$
\begin{aligned}
& \mathbb{P}_{4}=-\epsilon^{2} \oint_{S_{\infty}}\left(\partial^{I} \phi_{0}-\partial_{J} \mathfrak{z}^{I J}\right) d S_{I}+\mathrm{O}\left(\epsilon^{3}\right), \\
& \mathbb{P}_{I}=\epsilon^{2} \oint_{S_{\infty}}\left(\delta_{I}^{J} \partial_{K} \mathfrak{w}_{0}^{K}-\partial^{J} \mathfrak{w}_{0}^{I}-\partial_{I} \mathfrak{w}_{0}^{4 J}-\mathfrak{z}_{4}^{I J}\right) d S_{J}+\mathrm{O}\left(\epsilon^{3}\right) .
\end{aligned}
$$

Using the divergence theorem and (4.21), the energy-momentum expansions (4.27) and (4.28) simplify to

$$
\mathbb{P}_{4}=-\epsilon^{2} \int_{\mathbb{R}^{3}} \rho d x^{3}+\mathrm{O}\left(\epsilon^{3}\right) \quad \text { and } \quad \mathbb{P}_{I}=\mathrm{O}\left(\epsilon^{3}\right) .
$$

The proof of the corollary now follows from the above expansions and the definition

$$
m_{\mathrm{ADM}}=\frac{1}{\epsilon^{2}} \sqrt{\eta^{i j} \mathbb{P}_{i} \mathbb{P}_{j}}
$$

of the ADM mass.

\subsection{Uniform existence}

To prove local existence of solutions to (4.1) on a uniform time interval independent of $\epsilon$, we take the same approach as in [11] and use a non-local modification of (4.1). The modified system is constructed as follows. First, we replace $g(V)$ in (4.1) with

$$
g(V)=\left(-\delta_{4}^{i} \delta_{4}^{j} \chi_{\bar{R}} \rho(\alpha), 0, \ldots, 0\right)^{\mathrm{T}},
$$

and we define the Newtonian potential by

$$
\Delta \Phi=\chi_{\bar{R}} \rho .
$$

Next, we use the Newtonian potential to define a new combined gravitational-matter variable $W$ via the formula

$$
W=V-d \Phi,
$$


where

$$
d \Phi=\left(0, \delta_{4}^{i} \delta_{4}^{j} \partial_{J} \Phi(\alpha), 0,0,0\right) .
$$

Note that the transformation (4.32) leaves the matter variables unaffected. Consequently, we can define $W$ by

$$
W=\left(\mathfrak{u}_{4}^{i j}, W_{I}^{i j}, \delta \mathfrak{u}^{i j}, \alpha, w^{i}\right)^{\mathrm{T}},
$$

and treat $\Phi$ and $d \Phi$ as a function of $W$. To formulate the evolution equation entirely in terms of $W$, we need the time derivative of the $\Phi$ map. So we define

$$
\begin{aligned}
\dot{\Phi}\left(W, \epsilon U, \epsilon W, \epsilon^{2} U\right)=\Delta^{-1}( & \frac{2 n \chi_{\bar{R}} \alpha^{2 n-1}}{(4 K n(n+1))^{n}} \Pi\left(a ^ { 4 } ( \epsilon \mathfrak { u } , \epsilon \mathbf { w } ) ^ { - 1 } \left[a^{I}(\mathbf{w}, \epsilon \mathfrak{u}, \epsilon \mathbf{w}) \partial_{I} \mathbf{w}\right.\right. \\
& +b(\mathfrak{u}, \mathbf{w}, \epsilon \mathfrak{u}, \epsilon \mathbf{w})]))
\end{aligned}
$$

where $\Pi\left(\left(\alpha, w^{i}\right)^{\mathrm{T}}\right)=\alpha$ is a constant projection map. By construction, $\dot{\Phi}=\partial_{t} \Phi$ when evaluated on a solution of (4.1). To fit with the above notation, we also define

$$
d \dot{\Phi}=\left(0, \delta_{4}^{i} \delta_{4}^{j} \partial_{I} \dot{\Phi}, 0,0,0\right)^{\mathrm{T}} .
$$

Noting that

$$
b^{0}\left(\epsilon V, \epsilon^{2} U\right)=b^{0}\left(\epsilon W, \epsilon^{2} U\right) \quad \text { and } \quad b^{I}\left(V, \epsilon U, \epsilon V, \epsilon^{2}\right)=b^{I}\left(W, \epsilon U, \epsilon W, \epsilon^{2} U\right),
$$

we can write (4.1) as

$$
\begin{aligned}
b^{0}\left(\epsilon W, \epsilon^{2} U\right) \partial_{t} W= & \frac{1}{\epsilon} c^{I} \partial_{I} W+b^{I}\left(W, \epsilon U, \epsilon W, \epsilon^{2} U\right) \partial_{I} W \\
& +\mathcal{F}_{0}\left(W, \epsilon U, \epsilon W, \epsilon^{2} U\right)+\epsilon \mathcal{F}_{1}\left(W, \epsilon U, \epsilon W, \epsilon^{2} U\right)
\end{aligned}
$$

where

$$
\begin{aligned}
\mathcal{F}_{0}\left(W, \epsilon U, \epsilon W, \epsilon^{2} U\right)= & f_{0}\left(W+d \Phi(W), \epsilon U, \epsilon(W+d \Phi(W)), \epsilon^{2} U\right) \\
& -b^{0}\left(\epsilon W, \epsilon^{2} W\right) d \dot{\Phi}\left(W, \epsilon U, \epsilon W, \epsilon^{2} U\right) \\
& +b^{I}(W, \epsilon U, \epsilon W) \partial_{I} d \Phi(W)
\end{aligned}
$$

and

$$
\left.\mathcal{F}_{1}\left(W, \epsilon U, \epsilon W, \epsilon^{2} U\right)\right)=f_{1}\left(W+d \Phi(W), \epsilon U, \epsilon(W+d \Phi(W)), \epsilon^{2} U\right) .
$$


In the following Proposition, the constant $C_{\text {Sob }}$ is defined to be the $\epsilon$-independent in the weighted Sobolev inequality $\|\cdot\|_{W_{\eta, \epsilon}^{1, \infty}} \leq C_{\text {Sob }}\|\cdot\|_{H_{\eta, \epsilon}^{\ell}}$ which holds for $\ell>3 / 2+1$ and $0 \leq \epsilon \leq \epsilon_{0}$ (see Lemma A.7 in [10] for a proof).

Proposition 4.3. Suppose $-1<\delta<-1 / 2, \epsilon_{0}>0, s \in \mathbb{N}_{0}, R>0, K_{1}<$

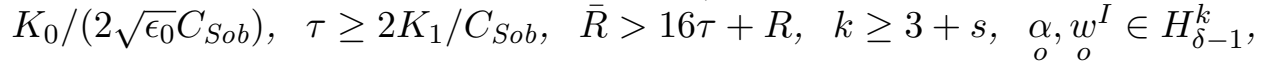
$\operatorname{supp} \underset{o}{\alpha} \subset B_{R}, \mathfrak{z}^{I J} \in H_{\delta}^{k+1}, \mathfrak{z}_{4}^{I J} \in H_{\delta-1}^{k}$. Let $\overline{\mathfrak{u}}_{\epsilon}^{i j}, \partial_{t} \overline{\mathfrak{u}}_{\epsilon}^{i j}$ and $w_{\epsilon}^{4}$ be the initial data constructed in Proposition 4.1, which, by choosing $\epsilon_{0} \leq 1$ small enough, satisfies

$$
\begin{gathered}
\left.\|\left(\epsilon \partial_{t} \overline{\mathfrak{u}}_{\epsilon}^{i j}, \partial_{I} \overline{\bar{u}}_{o}^{i j}-\delta_{4}^{i} \delta_{4}^{j} \partial_{I} \Delta^{-1} \underset{o}{\rho}, 0, \underset{o}{\alpha, w_{\epsilon}}\right)^{i}\right)^{\mathrm{T}} \|_{H_{\delta-1, \epsilon}^{k}} \leq K_{1}, \\
\text { and } \quad\left\|\overline{\mathfrak{u}}_{\epsilon}^{i j}\right\|_{H_{\delta}^{k+1}} \leq \frac{K_{0}}{\sqrt{\epsilon_{0}} C_{S o b}}
\end{gathered}
$$

for all $\epsilon \in\left(0, \epsilon_{0}\right]$. Then there exists a $T>0$ independent of $\epsilon \in\left(0, \epsilon_{0}\right]$, and maps

$$
W_{\epsilon}=\left(\mathfrak{u}_{4, \epsilon}^{i j}, W_{I, \epsilon}^{i j}, \delta \mathfrak{u}_{\epsilon}^{i j}, \alpha_{\epsilon}, w_{\epsilon}^{i}\right)^{\mathrm{T}} \in X_{T_{\epsilon}, s, k, \delta-1} \quad 0<\epsilon \leq \epsilon_{0}
$$

such that

(i) $T_{\epsilon}>T$ for $0 \leq \epsilon \leq \epsilon_{0}$,

(ii) $W_{\epsilon}$ is the unique solution to (4.36) with initial data

$$
\left.W_{\epsilon}(0)=\left(\epsilon \partial_{t} \overline{\mathfrak{u}}_{\epsilon}^{i j}, \partial_{I} \overline{\mathfrak{u}}_{o}^{i j}-\delta_{4}^{i} \delta_{4}^{j} \partial_{I} \Delta^{-1} \underset{o}{\rho}, \underset{o}{\alpha, w_{o}}\right)^{i}\right)^{\mathrm{T}}
$$

(iii)

$$
\left\|W_{\epsilon}(t)\right\|_{H_{\delta-1, \epsilon}^{k}} \leq 2 K_{1}, \quad \epsilon\left\|\partial_{t} W_{\epsilon}(t)\right\|_{H_{\delta-1, \epsilon}^{k-1}} \lesssim 1
$$

and

$$
\max \left\{\left\|\epsilon \overline{\mathfrak{u}}_{\epsilon}^{i j}(t)\right\|_{L^{\infty}},\left\|\epsilon \alpha_{\epsilon}(t)\right\|_{L^{\infty}},\left\|\epsilon w^{i}(t)\right\|_{L^{\infty}}\right\}<2 K_{0}
$$

for all $(t, \epsilon) \in[0, T] \times\left(0, \epsilon_{0}\right]$, 
(iv) for each $\epsilon \in\left(0, \epsilon_{0}\right]$, if

$$
\limsup _{t \nearrow T_{\epsilon}}\left\|W_{\epsilon}(t)\right\|_{W^{1, \infty}}<\infty
$$

and

$$
\sup _{0 \leq t<T_{\epsilon}}\left\{\left\|\epsilon \overline{\mathfrak{u}}_{\epsilon}^{i j}(t)\right\|_{L^{\infty}},\left\|\epsilon \alpha_{\epsilon}(t)\right\|_{L^{\infty}},\left\|\epsilon w^{i}(t)\right\|_{L^{\infty}}\right\}<2 K_{0},
$$

then the solution $W_{\epsilon}(t)$ can be uniquely extended for some time $T_{\epsilon}^{*}>T_{\epsilon}$,

(v) for any time $\tilde{T}_{\epsilon}$ which is strictly less than the maximal existence time and for which

$$
\sup _{0 \leq t \leq T_{\epsilon}}\left\{\left\|\epsilon \overline{\mathfrak{u}}_{\epsilon}^{i j}(t)\right\|_{L^{\infty}},\left\|\epsilon \alpha_{\epsilon}(t)\right\|_{L^{\infty}},\left\|\epsilon w^{i}(t)\right\|_{L^{\infty}}\right\}<2 K_{0}
$$

holds, the support of $\alpha_{\epsilon}$ satisfies

$$
\operatorname{supp} \alpha_{\epsilon}(t) \subset B_{\bar{R}_{\epsilon}} \quad \forall t \in\left[0, \tilde{T}_{\epsilon}\right]
$$

where $\bar{R}_{\epsilon}:=16 \sup _{0 \leq t \leq \tilde{T}_{\epsilon}}\left\|w_{\epsilon}^{I}(t)\right\|_{L^{\infty}}+R$,

(vi) $\operatorname{supp} \alpha_{\epsilon}(t) \subset B_{\bar{R}}$ for all $(t, \epsilon) \in[0, T] \times\left(0, \epsilon_{0}\right]$,

(vii) $\partial_{t} \overline{\mathfrak{u}}_{\epsilon}^{i j}=\epsilon^{-1} \overline{\mathfrak{u}}_{4, \epsilon}^{i j}$, and $\partial_{I} \overline{\bar{u}}_{\epsilon}^{i j}=W_{I, \epsilon}^{i j}+\delta_{4}^{i} \delta_{4}^{j} \partial_{I} \Phi\left(\alpha_{\epsilon}\right)$ where $\overline{\mathfrak{u}}_{\epsilon}^{i j}=\overline{\mathfrak{u}}_{\epsilon}^{i j}+$ $\epsilon^{-1} \delta \mathfrak{u}^{i j}$,

(viii) the triple $\left\{\overline{\mathfrak{u}}_{\epsilon}^{i j}, \alpha_{\epsilon}, w_{\epsilon}^{i}\right\}$ determines, via the formulas (1.7), (1.9), (2.4), and (2.5), a solution to the full Einstein-Euler system (1.1) in the harmonic gauge (2.16) on the spacetime region $D_{\epsilon}=\mathbb{R}^{3} \times[0, T]$, and

(ix) the conclusions (vii)-(viii) continue to hold on any region of the form $D_{\epsilon}=\mathbb{R}^{3} \times\left[0, \tilde{T}_{\epsilon}\right]$ provided supp $\alpha_{\epsilon}(t) \subset B_{\bar{R}}$ for all $0 \leq t \leq \tilde{T}_{\epsilon}$.

Proof. (i)-(vii): First we observe, that proof of statements (i)-(vii) follow from a slight modification of the proof of Proposition 3.4 in [11].

(viii)-(ix): Let $\psi_{\epsilon}$ satisfy the initial value problem

$$
\bar{v}^{k} \bar{\partial}_{k} \psi_{\epsilon}=0: \psi_{\epsilon}(0)=\chi_{3 \bar{R} / 2}(0)
$$

and define

$$
\tilde{\mathcal{N}}_{\epsilon}=\psi \mathcal{N}_{\epsilon}=\psi\left(\epsilon \bar{v}_{i} \bar{v}^{i}+\frac{1}{\epsilon}\right) .
$$


Next, we observe that (3.10) contracted with $\bar{v}^{i}$ yields

$$
\left(1-2 \epsilon \mathcal{N}_{\epsilon}\right) \bar{v}^{j} \bar{\partial}_{j} \mathcal{N}_{\epsilon}-\frac{\epsilon^{2} \alpha}{2 n h} \bar{v}^{j} \bar{\partial}_{j} \alpha \mathcal{N}_{\epsilon}+\epsilon\left(1-2 \epsilon \mathcal{N}_{\epsilon}\right)\left(\chi_{4 \bar{R}}-1\right) \bar{v}^{i} \bar{v}^{j} \bar{\Gamma}_{i j}^{k} \bar{v}_{k}=0 .
$$

Multiplying this equation by $\psi_{\epsilon}$ then gives

$$
\left(1-2 \epsilon \mathcal{N}_{\epsilon}\right) v b^{j} \bar{\partial}_{j} \tilde{\mathcal{N}}_{\epsilon}-\frac{\epsilon^{2} \alpha}{2 n h} \bar{v}^{j} \bar{\partial}_{j} \alpha \tilde{\mathcal{N}}_{\epsilon}+\epsilon\left(1-2 \epsilon \mathcal{N}_{\epsilon}\right) \psi_{\epsilon}\left(\chi_{4 \bar{R}}-1\right) \bar{v}^{i} \bar{v}^{j} \bar{\Gamma}_{i j}^{k} \bar{v}_{k}=0 .
$$

From statements (i)-(vii), we have that

$$
w_{\epsilon}^{i}(t) \in X_{T, s, k, \delta-1}
$$

and

$$
\left\|w^{i}(t)\right\|_{H^{k}} \lesssim\left\|w^{i}(t)\right\|_{H_{\delta-1, \epsilon}^{k}} \lesssim 1 \quad \forall(t, \epsilon) \in[0, T) \times(0, \epsilon] .
$$

Since

$$
\bar{v}^{j} \partial_{i}=\left(1+\epsilon w_{\epsilon}^{4}\right) \partial_{t}+w_{\epsilon}^{I} \partial_{I},
$$

we get from (4.41), (4.42), and the hyperbolic (4.39) that $\psi \in X_{T, s, k, \delta-1}$ and

$$
\left\|\psi_{\epsilon}(t)\right\|_{H^{k}} \lesssim 1 \quad \forall(t, \epsilon) \in[0, T) \times(0, \epsilon] .
$$

From the finite propagation speed property of hyperbolic equations, we conclude that there exists a time $T_{*} \in(0, T)$ such that

$$
\left.\psi_{\epsilon}(t)\right|_{B_{\bar{R}}}=1, \quad \text { and } \quad \operatorname{supp} \psi_{\epsilon}(t) \subset B_{4 \bar{R}} \quad \forall(t, \epsilon) \in\left[0, T_{*}\right) \times\left(0, \epsilon_{0}\right] .
$$

In particular, this implies that

$$
\mathcal{N}_{\epsilon}(t, x)=\tilde{\mathcal{N}}_{\epsilon}(t, x) \quad \forall(t, x, \epsilon) \in\left[0, T_{*}\right) \times B_{\bar{R}} \times\left(0, \epsilon_{0}\right],
$$

and

$$
\left(\chi_{4 \bar{R}}(x)-1\right) \psi_{\epsilon}(t, x)=0 \quad \forall(t, x, \epsilon) \in\left[0, T_{*}\right) \times \mathbb{R}^{3} \times\left(0, \epsilon_{0}\right] .
$$

Using (4.45), (4.40) reduces to

$$
\left(1-2 \epsilon \mathcal{N}_{\epsilon}\right) v b^{j} \bar{\partial}_{j} \tilde{\mathcal{N}}_{\epsilon}-\frac{\epsilon^{2} \alpha}{2 n h} \bar{v}^{j} \bar{\partial}_{j} \alpha \tilde{\mathcal{N}}_{\epsilon}=0
$$

for all $(t, x, \epsilon) \in\left[0, T_{*}\right) \times \mathbb{R}^{3} \times\left(0, \epsilon_{0}\right]$. But $\mathcal{N}_{\epsilon}(0)=0$ from the choice of initial data which implies that $\tilde{\mathcal{N}}_{\epsilon}(0)=0$. By the uniqueness of solutions to 
hyperbolic equations, we conclude that $\tilde{\mathcal{N}}_{\epsilon}(t, x)=0$ for all $(t, x, \epsilon) \in\left[0, T_{*}\right) \times$ $\mathbb{R}^{3} \times\left(0, \epsilon_{0}\right]$, and hence $\mathcal{N}_{\epsilon}(t, x)=0$ for all $(t, x, \epsilon) \in\left[0, T_{*}\right) \times B_{\mathbb{R}} \times\left(0, \epsilon_{0}\right]$. This implies that the fluid velocity normalization $\bar{v}^{i} \bar{v}_{i}=-1 / \epsilon^{2}$ is satisfied for all $(t, x, \epsilon) \in\left[0, T_{*}\right) \times B_{\mathbb{R}} \times\left(0, \epsilon_{0}\right]$. Using this and the fact that $\operatorname{supp} \alpha_{\epsilon}(t) \subset B_{\mathbb{R}}$ for all $(t, \epsilon) \in\left[0, T_{*}\right) \times\left(0, \epsilon_{0}\right]$, it is not difficult to verify from the evolution (3.9) to (3.10) that

$$
\begin{aligned}
\left\{\bar{v}^{4}(t, x)\right. & =1+\epsilon w_{\epsilon}^{4}(t, x), \bar{v}^{I}(t, x)=w_{\epsilon}^{I}(t, x), \\
\rho_{\epsilon}(t, x) & \left.:=(4 K n(n+1))^{-n} \alpha_{\epsilon}^{2 n}(t, x)\right\}
\end{aligned}
$$

satisfy the Euler (3.4) (or equivalently (3.1)) for all $(t, x, \epsilon) \in\left[0, T_{*}\right) \times \mathbb{R}^{3} \times$ $\left(0, \epsilon_{0}\right]$. With the Euler equations satisfied, the remainder of the proof follows as in the proof of Proposition 3.4 in [11].

\section{$5 \quad$ Limit equations}

In this section, we describe the limit equations that govern the gravitational and matter fields in the limit $\epsilon \searrow 0$. We show in the next section that solutions to these equations approximate the solutions to the full EinsteinEuler equations up to a remainder term that is of order $\epsilon$.

\subsection{Fluid limit equations}

The fluid limit equations are

$$
\begin{aligned}
\partial_{t} \tilde{\alpha} & =-\tilde{w}^{I} \partial_{I} \tilde{\alpha}-\frac{\tilde{\alpha}}{2 n} \partial_{I} \tilde{w}^{I}, \\
\partial_{t} \tilde{w}^{J} & =-\frac{\tilde{\alpha}}{2 n} \partial^{J} \tilde{\alpha}-\tilde{w}^{I} \partial_{I} \tilde{w}^{J}-\chi_{4} \partial^{J} \partial^{J}, \\
\Delta \tilde{\Phi} & =\tilde{\rho} \quad\left(\tilde{\rho}:=(4 K n(n+1))^{-n} \tilde{\alpha}^{2 n}\right) .
\end{aligned}
$$

Proposition 5.1. Let $k, s, \bar{R}, \delta, \underset{o}{\alpha}$, and $\underset{o}{w}$ be as in Proposition 4.3. Then there exists a maximal time $T_{0}^{M}>0$ and a unique solution

$$
\begin{gathered}
\tilde{\alpha}, \tilde{w}^{I} \in C^{0}\left(\left[0, T_{0}^{M}\right), H_{\delta-1}^{k}\right) \cap C^{1}\left(\left[0, T_{0}\right), H_{\delta-1}^{k-1}\right), \\
\tilde{\Phi} \in C^{0}\left(\left[0, T_{0}^{M}\right), H_{\delta}^{k+2}\right) \cap C^{1}\left(\left[0, T_{0}^{M}\right), H_{\delta}^{k+1}\right), \quad \partial_{t} \tilde{\Phi} \in C^{0}\left(\left[0, T_{0}^{M}\right), H_{\delta-1}^{k+1}\right)
\end{gathered}
$$


to (5.1)-(5.3) satisfying $\tilde{\alpha}(0)=\underset{o}{\alpha}$ and $\tilde{w}^{I}(0)=\underset{o}{w}$. Moreover,

$$
\begin{gathered}
\tilde{\alpha}, \tilde{w}^{I} \in X_{T_{0}^{M}, s, k, \delta-1}, \quad \tilde{\Phi} \in X_{T_{0}^{M}, s, k+2, \delta}, \\
\partial_{t} \tilde{\Phi}=-\partial_{I} \Delta^{-1}\left(\tilde{\rho} \tilde{w}^{I}\right) \in X_{T_{0}^{M}, s, k+1, \delta-1},
\end{gathered}
$$

and

$$
\operatorname{supp} \tilde{\alpha}(t) \subset B_{R(t)}
$$

where $R(t)=R+t \sup _{0 \leq s \leq t}\left\|\tilde{w}^{I}(s)\right\|_{L^{\infty}}$.

Proof. The proof follows from a trivial modification of the proof in Proposition 3.7 in [11].

Remark 5.2. Since $\bar{R}>R$, it is clear from Proposition 5.1 and the weighted Sobolev inequality (see Lemma A.7 in [10]) that there exists a time $T_{0} \in$ $\left(0, T_{M}\right)$ such that

$$
\operatorname{supp} \tilde{\alpha}(t) \subset B_{4 \bar{R}} \quad \text { for all } t \in\left[0, T_{0}\right] .
$$

In particular, this shows that

$$
\tilde{\rho}(t) \chi_{4 \bar{R}}=\tilde{\rho}(t) \quad \text { for all } t \in\left[0, T_{0}\right]
$$

which in turn implies that the pair $\left\{\tilde{\rho}(t), \tilde{w}^{I}(t)\right\}$ satisfies the Poisson-Euler (1.2) to (1.4) on the time interval $\left[0, T_{0}\right]$.

\subsection{Gravitational-limit equations}

The gravitational-limit equations are defined by

$$
\partial_{t} \mathbf{X}_{\epsilon}=\frac{1}{\epsilon} C^{I} \partial_{I} \mathbf{X}_{\epsilon}+\left(0,0, X_{4, \epsilon}^{i j}\right)^{\mathrm{T}},
$$

where

$$
\mathbf{X}_{\epsilon}=\left(X_{4, \epsilon}^{i j}, X_{I, \epsilon}^{i j}, X_{\epsilon}^{i j}\right)^{\mathrm{T}} .
$$


Proposition 5.3. Let $\delta, k, \underset{o}{\alpha}, \partial_{t} \overline{\mathfrak{u}}_{\epsilon}^{i j}, \partial_{I} \overline{\bar{u}}_{\epsilon}^{i j}$ be as in Proposition 4.3, and

$$
\mathbf{X}_{\epsilon}(0)=\left.\left(\epsilon \partial_{t} \overline{\mathfrak{u}}_{\epsilon}^{i j}, \partial_{I} \overline{\mathfrak{u}}_{\epsilon}^{i j}-\partial_{I} \Delta^{-1}\left(\delta_{4}^{i} \delta_{4}^{j} \rho\right), 0\right)^{\mathrm{T}}\right|_{\epsilon=0}
$$

Then there exists a unique solution

$$
\mathbf{X}_{\epsilon} \in C^{0}\left([0, \infty), H_{\delta-1}^{k}\right) \cap C^{1}\left([0, \infty), H_{\delta-1}^{k-1}\right)
$$

to (5.6) with initial data (5.7) that satisfies $\mathbf{X}_{\epsilon} \in X_{\infty, s, k, \delta-1}$ and the estimates:

(i)

$$
\begin{gathered}
\left\|\mathbf{X}_{\epsilon}(t)\right\|_{H_{\delta-1, \epsilon}^{k}}+\epsilon\left\|\partial_{t} \mathbf{X}_{\epsilon}(t)\right\|_{H_{\delta-1, \epsilon}^{k-1}} \lesssim e^{C t} \\
\left\|X_{\epsilon}^{i j}(t)\right\|_{L_{\delta, \epsilon}^{\infty}}+\left\|D X_{\epsilon}^{i j}(t)\right\|_{H_{\delta-1, \epsilon}^{k-1}} \lesssim e^{C t} \epsilon,
\end{gathered}
$$

for all $(t, \epsilon) \in[0, \infty) \times\left(0, \epsilon_{0}\right]$ and some fixed constant $C>0$, and

(ii) for any $\Lambda>0$,

$$
\begin{aligned}
& \left\|X_{I, \epsilon}^{i j}(t)\right\|_{W^{\ell, \infty}\left(B_{\Lambda}\left(\mathbb{R}^{3}\right)\right.}+\left\|X_{4, \epsilon}^{i j}(t)\right\|_{W^{\ell, \infty}\left(B_{\Lambda}\left(\mathbb{R}^{3}\right)\right)} \\
& \lesssim \frac{\epsilon^{3 / 2} \sqrt{\Lambda+1}}{(\epsilon+t)^{3 / 2}} \quad 0 \leq \ell<k-3 / 2 \\
& \text { for all }(t, \epsilon) \in[0, \infty) \times\left(0, \epsilon_{0}\right] \text {. }
\end{aligned}
$$

Proof. Since $-1<\delta<-1 / 2$, it follows from Lemma A.11 of [10] and Proposition 4.1 that

$$
\left\|\mathbf{X}_{\epsilon}(0)\right\|_{H_{\delta-1}^{k}, \epsilon} \lesssim\left\|\mathbf{X}_{\epsilon}(0)\right\|_{H_{\delta-1}^{k}} \lesssim 1
$$

This inequality together with the weighted energy estimates (see Lemma 7.1 in [10]) gives

$$
\left\|\mathbf{X}_{\epsilon}(t)\right\|_{H_{\delta-1}^{k}} \lesssim e^{C t}\left\|\mathbf{X}_{\epsilon}(0)\right\|_{H_{\delta-1, \epsilon}^{k}} \lesssim e^{C t}
$$

for some fixed positive constant $C$. 
From the evolution (5.6) and the choice of initial data, we see that

$$
\partial_{t}\left(\partial_{I} X_{J, \epsilon}^{i j}(t)-\partial_{J} X_{I, \epsilon}^{i j}\right)=0 \quad \text { and } \quad \partial_{I} X_{J, \epsilon}^{i j}(0)-\partial_{J} X_{I, \epsilon}^{i j}=0
$$

which implies that

$$
\partial_{I} X_{J, \epsilon}^{i j}(t)-\partial_{I} X_{J, \epsilon}^{i j}(t)=0
$$

Also, it is not difficult to show that

$$
\partial_{t}\left(\partial_{I} X_{\epsilon}^{i j}-\epsilon X_{I, \epsilon}^{i j}\right)=0
$$

follows from (5.6). Combining (5.10) and (5.11) then yields

$$
\partial_{I} X_{\epsilon}^{i j}(t)=\epsilon\left(X_{I, \epsilon}^{i j}(t)-X_{I, \epsilon}^{i j}(0)\right)
$$

Next, we note that

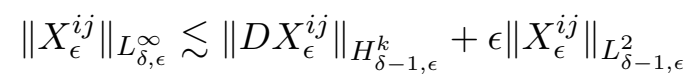

follows from the weighted Sobolev inequalities (see Lemma A.7 in [10]). Collecting the estimates (5.9), (5.12), and (5.13), we arrive at

$$
\left\|X_{\epsilon}^{i j}(t)\right\|_{L_{\delta, \epsilon}^{\infty}}+\left\|D X_{\epsilon}^{i j}\right\|_{H_{\delta-1, \epsilon}^{k}} \lesssim e^{C t} \epsilon
$$

To prove the last two estimates for $X_{I, \epsilon}^{i j}$ and $X_{I, \epsilon}^{4}$, we observe that $X_{I, \epsilon}^{i j}$ and $X_{I, \epsilon}^{4}$ satisfy the wave equations

$$
\epsilon^{2} \partial_{t}^{2} X_{I, \epsilon}^{i j}-\Delta X_{I, \epsilon}^{i j}=0 \quad \text { and } \quad \epsilon^{2} \partial_{t}^{2} X_{4, \epsilon}^{i j}-\Delta X_{I, \epsilon}^{i j}=0 .
$$

Since the initial data for these equations satisfy (5.8) and $-1<\delta<-1 / 2$, we can apply the weighted dispersive estimates from Theorem 1.1 in [4] 
to obtain

$$
\left|D_{x}^{\ell} X_{I, \epsilon}^{i j}(t, x)\right| \lesssim \frac{\left\|X_{I, \epsilon}^{i j}(0)\right\|_{H_{\delta-1}^{k}}+\left\|\partial_{I} X_{4, \epsilon}^{i j}(0)\right\|_{H_{\delta-2}^{k-1}}}{(1+t / \epsilon+|x|) \sqrt{|1+| t / \epsilon-|x| \mid}}
$$

and

$$
\left|D_{x}^{\ell} X_{4, \epsilon}^{i j}(t, x)\right| \lesssim \frac{\left\|X_{4, \epsilon}^{i j}(0)\right\|_{H_{\delta-1}^{k}}+\left\|\partial^{I} X_{I, \epsilon}^{i j}(0)\right\|_{H_{\delta-2}^{k-1}}}{(1+t / \epsilon+|x|) \sqrt{|1+| t / \epsilon-|x| \mid}}
$$

for $0 \leq \ell<k-3 / 2$. But

$$
\begin{aligned}
& 1+|t / \epsilon| \leq 1+|t / \epsilon-| x|+| x|| \leq 1+\Lambda+|t / \epsilon-| x|| \\
& \quad \leq(\Lambda+1)(1+|t / \epsilon-| x||)
\end{aligned}
$$

for $|x| \leq \Lambda$, and so the inequalities (5.15) and (5.16) imply that

$$
\left\|X_{I, \epsilon}^{i j}(t)\right\|_{W^{\ell, \infty}\left(B_{\Lambda}\left(\mathbb{R}^{3}\right)\right)}+\left\|X_{4, \epsilon}^{i j}(t)\right\|_{W^{\ell, \infty}\left(B_{\Lambda}\left(\mathbb{R}^{3}\right)\right)} \lesssim \frac{\epsilon^{3 / 2} \sqrt{\Lambda+1}}{(\epsilon+t)^{3 / 2}}
$$

for $0 \leq \ell<k-3 / 2$.

Remark 5.4. From the initial value problem (5.6)-(5.7) (see (5.12)) and Propositions 4.1 and 5.1 , it is not difficult to verify that

$$
\begin{aligned}
\tilde{\overline{\mathfrak{u}}}_{\epsilon}^{i j}:= & \frac{1}{\epsilon} X_{\epsilon}^{i j}+\left(\delta_{I}^{i} \delta_{J}^{j} \mathfrak{z}^{I J}-2 \Delta^{-1} \partial_{I} \mathfrak{z}_{4}^{I J} \delta_{4}^{(i} \delta_{J}^{j)}+\delta_{4}^{i} \delta_{4}^{j} \Delta^{-1}\left(\rho_{o}+\partial_{I J}^{2} \mathfrak{z}^{I J}\right)\right) \\
& +(\tilde{\Phi}(t)-\tilde{\Phi}(0)) \delta_{4}^{i} \delta_{4}^{j}
\end{aligned}
$$

satisfies the identities

$$
\partial_{t} \tilde{\overline{\mathfrak{u}}}_{\epsilon}^{i j}=\frac{1}{\epsilon} X_{4, \epsilon}^{i j}+\delta_{4}^{i} \delta_{4}^{j} \partial_{t} \tilde{\Phi}, \quad \partial_{I} \tilde{\overline{\mathcal{u}}}_{\epsilon}^{i j}=X_{I, \epsilon}^{i j}+\delta_{4}^{i} \delta_{4}^{j} \partial_{I} \tilde{\Phi}
$$

and the wave equation

$$
\epsilon^{2} \partial_{t}^{2} \tilde{\overline{\mathfrak{u}}}_{\epsilon}^{i j}-\Delta \tilde{\overline{\mathfrak{u}}}_{\epsilon}^{i j}=-\delta_{4}^{i} \delta_{4}^{j} \tilde{\rho}+\delta_{4}^{i} \delta_{4}^{j} \epsilon^{2} \partial_{t}^{2} \tilde{\Phi}
$$


with initial conditions

$$
\begin{gathered}
\left.\tilde{\tilde{\mathfrak{u}}}_{\epsilon}^{i j}\right|_{t=0}=\delta_{I}^{i} \delta_{J}^{j} \mathfrak{z}^{I J}-2 \Delta^{-1} \partial_{I} \mathfrak{z}_{4}^{I J} \delta_{4}^{(i} \delta_{J}^{j)}+\delta_{4}^{i} \delta_{4}^{j}\left(\left.\tilde{\Phi}\right|_{t=0}+\Delta^{-1} \partial_{I J}^{2} \mathfrak{z}^{I J}\right) \\
\left.\partial_{t} \tilde{\overline{\mathfrak{u}}}_{\epsilon}^{i j}\right|_{t=0}=\frac{1}{\epsilon}\left(\delta_{I}^{i} \delta_{J}^{j} \mathfrak{z}_{4}^{I J}-2 \partial_{I} \mathfrak{z}^{I J} \delta_{4}^{(i} \delta_{J}^{j)}+\delta_{4}^{i} \delta_{4}^{j} \Delta^{-1} \partial_{I J}^{2} \mathfrak{z}_{4}^{I J}\right)+\left.\delta_{4}^{i} \delta_{4}^{j} \partial_{t} \tilde{\Phi}\right|_{t=0} .
\end{gathered}
$$

\subsection{The combined system}

Collecting the fluid and gravitation limit variables into a single vector

$$
Y_{\epsilon}=\left(\mathbf{X}_{\epsilon}, \tilde{\alpha}, \tilde{w}^{I}, \tilde{w}^{4}\right)^{\mathrm{T}},
$$

we can write the combined gravitational-fluid limit equations in the following form:

$$
\partial_{t} Y_{\epsilon}=\frac{1}{\epsilon} c^{I} \partial_{I} Y+\tilde{b}_{0}^{I} \partial_{I} Y_{\epsilon}+\tilde{\mathcal{F}}_{0}+c^{I} \partial_{I} \omega
$$

where

$$
\begin{gathered}
\tilde{b}_{0}^{I}=\left(\begin{array}{cc}
0 & 0 \\
0 & \tilde{a}^{I}
\end{array}\right), \quad \tilde{a}^{I}=\left(\begin{array}{cc}
-\tilde{w}^{I} & -\frac{\tilde{\alpha}}{2 n} \delta_{j}^{I} \\
-\frac{\tilde{\alpha}}{2 n} \delta_{i}^{I} & -\delta_{i j} \tilde{w}^{I}
\end{array}\right), \\
\omega=\left(\omega_{4}^{i j}, \omega_{I}^{i j}, 0,0,0,0\right)^{\mathrm{T}}, \quad \omega_{4}^{i j}=\partial_{t} \tilde{\Phi} \delta_{4}^{i} \delta_{4}^{j}, \quad \omega_{I}^{i j}=\partial_{I} \Delta^{-1}\left(2 \rho \tilde{w}^{J} \delta_{J}^{(i} \delta_{4}^{j j}\right),
\end{gathered}
$$

and

$$
\tilde{\mathcal{F}}_{0}=\left(-2 \rho \tilde{w}^{J} \delta_{J}^{(i} \delta_{4}^{j)},-\partial_{I} \partial_{t} \tilde{\Phi} \delta_{4}^{i} \delta_{j}^{4}, X_{4}^{i j}, 0,-\chi_{4} \bar{R}^{I} \tilde{\Phi}, 0\right)^{\mathrm{T}} .
$$

\section{The fast Newtonian limit}

We begin by defining the error $Z_{\epsilon}$ between the limit $Y_{\epsilon}$ and the full solution $W_{\epsilon}$ by

$$
W_{\epsilon}=Y_{\epsilon}+\epsilon\left(\omega_{\epsilon}+Z_{\epsilon}\right) .
$$


Next, we let

$$
\tilde{\mathcal{F}}=\mathcal{F}_{0}\left(Y_{\epsilon}, 0,0,0\right), \quad \text { and } \quad \tilde{b}^{I}=b^{I}\left(Y_{\epsilon}, 0,0,0\right)
$$

and observe that

$$
\tilde{\mathcal{F}}=\tilde{\mathcal{F}}_{0}+\epsilon \tilde{\mathcal{F}}_{1}, \quad \text { and } \quad \tilde{b}^{I}=\tilde{b}_{0}^{I}+\epsilon \tilde{b}_{1}^{I},
$$

where

$$
\tilde{b}_{1}^{I}=\frac{1}{\epsilon}\left(\begin{array}{cc}
\tilde{A}^{I} & 0 \\
0 & 0
\end{array}\right), \quad \tilde{A}^{I}=\left(\begin{array}{ccc}
8 X_{\epsilon}^{4 I} & 4 X_{\epsilon}^{I J} & 0 \\
4 X_{\epsilon}^{I J} & 0 & 0 \\
0 & 0 & 0
\end{array}\right)
$$

and

$$
\tilde{\mathcal{F}}_{1}=\frac{1}{\epsilon}\left(4\left(X_{\epsilon}^{I J} \partial_{I J}^{2} \tilde{\Phi}-\tilde{\rho} \eta_{i j} X_{\epsilon}^{i j}\right) \delta_{4}^{i} \delta_{4}^{j}, 0,0,0, \tilde{\mathcal{F}}_{1}^{i}\right)^{\mathrm{T}}
$$

with

$$
\tilde{\mathcal{F}}_{1}^{I}=-\chi_{4 \bar{R}}\left[\delta^{I J}\left(X_{J, \epsilon}^{44}+\delta_{K L} X_{I, \epsilon}^{K L}\right)+4 X_{4}^{J 4}\right]
$$

and

$$
\tilde{\mathcal{F}}_{1}^{4}=-\chi_{4 \bar{R}}\left(X_{4, \epsilon}^{44}+\delta_{K L} X_{4, \epsilon}^{K L}\right)
$$

Now, let $T_{*}=\min \left\{T_{0}, T\right\}$ where $T$ and $T_{0}$ are as defined in Propositions 4.3 and Remark 5.2, respectively. Then by Propositions 4.3, 5.1, and 5.2 , for any $\epsilon \in\left(0, \epsilon_{0}\right]$, the error $Z_{\epsilon}(t)$ (see (4.36), (5.23), and $(6.1)-(6.3)$ ) satisfies the initial value problem

$$
\begin{aligned}
b_{\epsilon}^{0} \partial_{t} Z_{\epsilon} & =\frac{1}{\epsilon} c^{I} \partial_{I} Z_{\epsilon}+b_{\epsilon}^{I} \partial_{I} Z_{\epsilon}+\mathcal{R}_{\epsilon}, \\
Z_{\epsilon}(0) & =\frac{1}{\epsilon}\left(W_{\epsilon}(0)-Y_{\epsilon}(0)\right)-\omega(0),
\end{aligned}
$$

on the interval $0 \leq t \leq T_{*}$, where

$$
\begin{gathered}
b_{\epsilon}^{0}=b^{0}\left(\epsilon W_{\epsilon}, \epsilon^{2} U_{\epsilon}\right), \quad b_{\epsilon}^{I}=b^{I}\left(W_{\epsilon}, \epsilon U_{\epsilon}, \epsilon W_{\epsilon}, \epsilon^{2} U_{\epsilon}\right), \\
\mathcal{F}_{\epsilon}=\mathcal{F}_{0}\left(W_{\epsilon}, \epsilon U_{\epsilon}, \epsilon W_{\epsilon}, \epsilon^{2} U_{\epsilon}\right)+\epsilon \mathcal{F}_{1}\left(W_{\epsilon}, \epsilon U_{\epsilon}, \epsilon W_{\epsilon}, \epsilon^{2} U_{\epsilon}\right),
\end{gathered}
$$


and

$$
\mathcal{R}_{\epsilon}=b_{\epsilon}^{I} \partial_{I} \omega-b_{\epsilon}^{0} \partial_{t} \omega+\frac{b_{\epsilon}^{I}-\tilde{b}^{I}}{\epsilon} \partial_{I} Y+\frac{\mathcal{F}_{\epsilon}-\tilde{\mathcal{F}}}{\epsilon}+\frac{b_{\epsilon}^{0}-\mathbb{I}}{\epsilon^{2}} \epsilon \partial_{t} Y+\tilde{b}_{1}^{I} \partial_{I} Y+\tilde{\mathcal{F}}_{1} .
$$

Proposition 6.1. Let $\delta, k, s \geq 2, T$, and $W_{\epsilon}(t)$ be as in Proposition 4.3, $T_{0}$ as in Remark 5.2, $Y_{\epsilon}(t)$ as defined by (5.22), and $T_{*}=\min \left\{T_{0}, T\right\}$. Then for $\epsilon_{0}>0$ small enough

$$
\left\|W_{\epsilon}(t)-Y_{\epsilon}(t)\right\|_{H_{\delta-1, \epsilon}^{k-2}} \lesssim \epsilon
$$

for all $(t, \epsilon) \in\left[0, T_{*}\right) \times\left(0, \epsilon_{0}\right]$.

Proof. By Propositions 4.1, 4.3, and 5.1, there exists a positive constant $C_{0}$ such that

$$
\left\|Z_{\epsilon}(0)\right\|_{H_{\delta-1}^{k}} \leq C_{0} \quad \text { for all } \epsilon \in\left(0, \epsilon_{0}\right] .
$$

Next, choosing $\epsilon_{0}$ small enough, it follows directly from Propositions 3.5 and 3.6 of [11] and Propositions 5.1 and 5.3 of the previous section that

$$
\left\|b_{\epsilon}^{I} \partial_{I} \omega-b_{\epsilon}^{0} \partial_{t} \omega+\frac{b_{\epsilon}^{I}-\tilde{b}^{I}}{\epsilon} \partial_{I} Y_{\epsilon}+\frac{\mathcal{F}_{\epsilon}-\tilde{F}_{0}}{\epsilon}\right\|_{H_{\delta-1, \epsilon}^{k-2}} \lesssim 1+\left\|Z_{\epsilon}\right\|_{H_{\delta-1, \epsilon}^{k-2}}
$$

for all $(t, \epsilon) \in\left[0, T_{*}\right) \times\left(0, \epsilon_{0}\right]$ provided $\left\|Z_{\epsilon}(t)\right\|_{H_{\delta-1, \epsilon}^{k-2}} \leq 2 C_{0} / \epsilon$. Also, from Lemmas A.1 and A.4 of [10], and Proposition 5.1 and 5.3, we see that

$$
\left\|\frac{b_{\epsilon}^{0}-\mathbb{I}}{\epsilon^{2}} \epsilon \partial_{t} Y_{\epsilon}+\tilde{b}_{1}^{I} \partial_{I} Y_{\epsilon}+\tilde{\mathcal{F}}_{1}\right\|_{H_{\delta-1, \epsilon}^{k-2}} \lesssim 1+\frac{\sqrt{\epsilon}}{(\epsilon+t)^{3 / 2}}
$$

for all $(t, \epsilon) \in\left[0, T_{*}\right) \times\left(0, \epsilon_{0}\right]$.

Defining the energy norm

$$
\|\cdot\|_{k-2, \delta, \epsilon}:=\sum_{|\alpha| \leq k-2}\left\langle D_{x}^{\alpha}(\cdot) \mid b_{\epsilon}^{0} D_{x}^{\alpha}(\cdot)\right\rangle,
$$

we see via Proposition 4.3 that

$$
\|\cdot\|_{H_{\delta-1, \epsilon}^{k-2}} \lesssim\|\cdot\|\left\|_{k-2, \delta-1, \epsilon} \lesssim\right\| \cdot \|_{H_{\delta-1, \epsilon}^{k-2}}
$$

uniformly for $(t, \epsilon) \in\left[0, T_{*}\right) \times\left(0, \epsilon_{0}\right]$. Setting $\mathcal{Z}_{\epsilon}=\epsilon Z_{\epsilon}$, the evolution (6.6) and the weighted energy estimates (see the proof of Theorem B.1 in [11]) in 
conjunction with Proposition 4.3 and the estimates (6.12)-(6.13) show that there exists a fixed constant $C_{1}>0$ such that

$$
\frac{d}{d t}\left\|\mathcal{Z}_{\epsilon}(t)\right\|_{k-2, \delta-1, \epsilon} \leq C_{1}\left(\left\|\mathcal{Z}_{\epsilon}(t)\right\|_{k-2, \delta-1, \epsilon}+\epsilon+\left(\frac{\epsilon}{\epsilon+t}\right)^{3 / 2}\right),
$$

for all $t$ such that $\left\|\mathcal{Z}_{\epsilon}(t)\right\|_{k-2, \delta-1, \epsilon} \leq 2 C_{0}$. Gronwall's inequality and (6.11) then show that

$$
\begin{aligned}
\left\|\mathcal{Z}_{\epsilon}(t)\right\|_{k-2, \delta-1, \epsilon} & \leq e^{C_{1} t} C_{0} \epsilon+\epsilon \int_{0}^{t} e^{C_{1}(t-s)} d s+\epsilon^{3 / 2} \int_{0}^{t} \frac{e^{C_{1}(t-s)}}{(\epsilon+s)^{3 / 2}} d s \\
& \leq \epsilon e^{C_{1} t}\left(C_{0}+1+\sqrt{\epsilon} \int_{0}^{t} \frac{1}{(\epsilon+s)^{3 / 2}} d s\right) \\
& \leq \epsilon e^{C_{1} t}\left(C_{0}+3\right),
\end{aligned}
$$

again for all $t$ such that $\left\|\mathcal{Z}_{\epsilon}(t)\right\|_{k-2, \delta-1, \epsilon} \leq 2 C_{0}$. Therefore choosing $\epsilon_{0}>0$ small enough we obtain

$$
\left\|Z_{\epsilon}(t)\right\|_{H_{\delta-1, \epsilon}^{k-2}} \lesssim 1
$$

for all $(t, \epsilon) \in\left[0, T_{*}\right) \times\left(0, \epsilon_{0}\right]$, and the proof is complete.

We are now ready to prove the main theorem.

Proof of Theorem 1.1.

(i): Since the ADM mass is conserved, statement (i) follows directly from Corollary 4.2 and Proposition 4.3.

(ii)-(iv): From the definition of $W_{\epsilon}$ and $Y_{\epsilon}$, we have

$$
\begin{aligned}
& \left\|\alpha_{\epsilon}(t)-\tilde{\alpha}(t)\right\|_{H_{\delta-1, \epsilon}^{k-2}}+\left\|w_{\epsilon}^{I}(t)-\tilde{w}^{I}(t)\right\|_{H_{\delta-1, \epsilon}^{k-2}}+\left\|w_{\epsilon}^{4}(t)\right\|_{H_{\delta-1, \epsilon}^{k-2}} \\
& \quad \leq\left\|W_{\epsilon}(t)-Y_{\epsilon}(t)\right\|_{H_{\delta-1, \epsilon}^{k-2}},
\end{aligned}
$$

and hence, by Proposition 6.1,

$$
\left\|\alpha_{\epsilon}(t)-\tilde{\alpha}(t)\right\|_{H_{\delta-1, \epsilon}^{k-2}}+\left\|w_{\epsilon}^{I}(t)-\tilde{w}^{I}(t)\right\|_{H_{\delta-1, \epsilon}^{k-2}}+\left\|w_{\epsilon}^{4}(t)\right\|_{H_{\delta-1, \epsilon}^{k-2}} \lesssim \epsilon
$$

for all $(t, \epsilon) \in\left[0, T_{*}\right) \times\left(0, \epsilon_{0}\right]$. Also by the weighted multiplication Lemma (see Lemma A.8 in [10]) and Propositions 4.3 and 5.1, we have

$$
\left\|\tilde{\rho}_{\epsilon}(t)-\tilde{\rho}_{\epsilon}(t)\right\|_{H_{\delta-1, \epsilon}^{k-2}} \lesssim\left\|\alpha_{\epsilon}(t)-\tilde{\alpha}(t)\right\|_{H_{\delta-1, \epsilon}^{k-2}}
$$


for all $t \in\left[0, T_{*}\right) \times\left(0, \epsilon_{0}\right]$, while

$$
\begin{aligned}
& \left\|\rho_{\epsilon}(t)-\tilde{\rho}(t)\right\|_{H^{k-2}}+\left\|w_{\epsilon}^{I}(t)-\tilde{w}^{I}(t)\right\|_{H^{k-2}}+\left\|w_{\epsilon}^{4}(t)\right\|_{H^{k-2}} \\
& \quad \lesssim\left\|\rho_{\epsilon}(t)-\tilde{\rho}(t)\right\|_{H_{\delta-1, \epsilon}^{k-2}}+\| w_{\epsilon}^{I}(t) \\
& \quad-\tilde{w}^{I}(t)\left\|_{H_{\delta-1, \epsilon}^{k-2}}+\right\| w_{\epsilon}^{4}(t) \|_{H_{\delta-1, \epsilon}^{k-2}}
\end{aligned}
$$

is a consequence of Lemma A.11 and (A.24) of [10]. Combining the inequalities (6.14)-(6.16), we arrive at

$$
\left\|\rho_{\epsilon}(t)-\tilde{\rho}(t)\right\|_{H^{k-2}}+\left\|w_{\epsilon}^{I}(t)-\tilde{w}^{I}(t)\right\|_{H^{k-2}}+\left\|w_{\epsilon}^{4}(t)\right\|_{H^{k-2}} \lesssim \epsilon
$$

for all $t \in\left[0, T_{*}\right) \times\left(0, \epsilon_{0}\right]$.

Next, we observe that

$$
\left\|\partial_{I} \Phi_{\epsilon}(t)-\partial_{I} \tilde{\Phi}(t)\right\|_{H_{\delta-1, \epsilon}^{k-2}}+\left\|\epsilon \partial_{t} \Phi_{\epsilon}(t)\right\|_{H_{\delta-1, \epsilon}^{k-2}} \lesssim \epsilon
$$

for all $(t, \epsilon) \in\left[0, T_{*}\right) \times\left(0, \epsilon_{0}\right]$ by Propositions 4.3 and 6.1 , and Lemmas 3.2 and 3.3 of [11]. From the above estimate, the identities $W_{I, \epsilon}^{i j}=\partial_{I} \overline{\mathfrak{u}}_{\epsilon}^{i j}+$ $\delta_{4}^{i} \delta_{4}^{j} \partial_{I} \Phi_{\epsilon}$ and $\mathfrak{u}_{4, \epsilon}^{i j}=\epsilon \partial_{t} \overline{\mathfrak{u}}_{\epsilon}^{i j}$ (see Proposition 4.3), and the relations (5.17) to (5.18), we get

$$
\begin{aligned}
& \left\|\partial_{I} \overline{\mathfrak{u}}_{\epsilon}^{i j}(t)-\partial_{I} \tilde{\tilde{\mathfrak{u}}}_{\epsilon}^{i j}(t)\right\|_{H_{\delta-1, \epsilon}^{k-2}}+\left\|\epsilon \partial_{t} \overline{\mathfrak{u}}_{\epsilon}^{i j}(t)-\epsilon \partial_{t} \tilde{\overline{\mathfrak{u}}}_{\epsilon}^{i j}(t)\right\|_{H_{\delta-1, \epsilon}^{k-2}} \\
& \quad \lesssim\left\|W_{I, \epsilon}^{i j}(t)-X_{I, \epsilon}^{i j}(t)\right\|_{H_{\delta-1, \epsilon}^{k-2}}+\left\|\mathfrak{u}_{4, \epsilon}^{i j}(t)-X_{4, \epsilon}^{i j}(t)\right\|_{H_{\delta-1, \epsilon}^{k-2}}+\epsilon \\
& \quad \lesssim\left\|W_{\epsilon}(t)-Y_{\epsilon}(t)\right\|_{H_{\delta-1, \epsilon}^{k-2}}+\epsilon
\end{aligned}
$$

and hence, by Proposition 6.1 and Lemma A.7 of [10],

$$
\begin{aligned}
& \left\|\overline{\bar{u}}_{\epsilon}^{i j}(t)-\tilde{\overline{\mathfrak{u}}}_{\epsilon}^{i j}(t)\right\|_{L_{\delta, \epsilon}^{6}}+\left\|\partial_{I} \overline{\bar{u}}_{\epsilon}^{i j}(t)-\partial_{I} \tilde{\overline{\mathfrak{u}}}_{\epsilon}^{i j}(t)\right\|_{H_{\delta-1, \epsilon}^{k-2}} \\
& \quad+\left\|\epsilon \partial_{t} \overline{\bar{u}}_{\epsilon}^{i j}(t)-\epsilon \partial_{t} \tilde{\overline{\mathfrak{u}}}_{\epsilon}^{i j}(t)\right\|_{H_{\delta-1, \epsilon}^{k-2}} \lesssim \epsilon
\end{aligned}
$$

for all $(t, \epsilon) \in\left[0, T_{*}\right) \times\left(0, \epsilon_{0}\right]$. Finally, it follows from Lemma A.11 and (A.24) of [10], and the above estimate that

$$
\begin{aligned}
& \left\|\overline{\mathfrak{u}}_{\epsilon}^{i j}(t)-\tilde{\tilde{\mathfrak{u}}}_{\epsilon}^{i j}(t)\right\|_{L^{6}}+\left\|\partial_{I} \overline{\mathfrak{u}}_{\epsilon}^{i j}(t)-\partial_{I} \tilde{\overline{\mathfrak{u}}}_{\epsilon}^{i j}(t)\right\|_{H^{k-2}} \\
& \quad+\left\|\epsilon \partial_{t} \overline{\overline{\mathfrak{u}}}_{\epsilon}^{i j}(t)-\epsilon \partial_{t} \tilde{\overline{\mathfrak{u}}}_{\epsilon}^{i j}(t)\right\|_{H^{k-2}} \lesssim \epsilon
\end{aligned}
$$

for all $(t, \epsilon) \in\left[0, T_{*}\right) \times\left(0, \epsilon_{0}\right]$. This completes the proof. 


\section{References}

[1] R. Bartnik, Phase space for the Einstein equations, Commun. Anal. Geom. 13 (2005), 845-885.

[2] U. Brauer and L. Karp, Local existence of classical solutions for the Einstein-Euler system using weighted Sobolev spaces of fractional order, C. R. Acad. Sci. Paris, Ser. I 345 (2007), 49-54.

[3] G. Browning and H.O. Kreiss, Problems with different time scales for nonlinear partial differential equations, SIAM J. Appl. Math. 42 (1982), 704-718.

[4] P. D'Ancona, V. Georgiev and H. Kubo, Weighted decay estimates for the wave equation, J. Differ. Equ. 177 (2001), 146-208.

[5] H. Isozaki, Wave operators and the incompressible limit of the compressible Euler equations, Comm. Math. Phys. 110 (1987), 519-524.

[6] S. Klainerman and A. Majda, Compressible and incompressible fluids, Comm. Pure Appl. Math. 35 (1982), 629-651.

[7] H.O. Kreiss, Problems with different time scales for partial differential equations, Comm. Pure Appl. Math. 33 (1980), 399-439.

[8] M. Lottermoser, A convergent post-Newtonian approximation for the constraints in general relativity, Ann. Inst. Henri Poincaré 57 (1992), 279-317.

[9] T. Makino, On a Local Existence Theorem for the Evolution Equation of Gaseous Stars, in 'Patterns and waves', eds. T. Nishida, M. Mimura and H. Fujii, North-Holland, Amsterdam, 1986.

[10] T.A. Oliynyk, The Newtonian limit for perfect fluids, Comm. Math. Phys. 276 (2007), 131-188.

[11] T.A. Oliynyk, Post-Newtonian expansions for perfect fluids, Comm. Math. Phys. 288 (2009), 847-886.

[12] A.D. Rendall, The initial value problem for a class of general relativistic fluid bodies, J. Math. Phys. 33 (1992), 1047-1053.

[13] A.D. Rendall, The Newtonian limit for asymptotically flat solutions of the Vlasov-Einstein system, Comm. Math. Phys. 163 (1994), 89-112.

[14] S. Schochet, Symmetric hyperbolic systems with a large parameter, Comm. Partial Differ. Equ. 11 (1986), 1627-1651.

[15] S. Schochet, Asymptotics for symmetric hyperbolic systems with a large parameter, J. Differ. Equ. 75 (1988), 1-27.

[16] S. Ukai, The incompressible limit and the initial layer of the compressible Euler equations, J. Math. Kyoto Univ. 26 (1986), 323-331. 
